\title{
Tight coupling expansion and fully inhomogeneous magnetic fields
}

\author{
Massimo Giovannini* \\ Centro "Enrico Fermi", Compendio del Viminale, Via Panisperna 89/A, 00184 Rome, Italy \\ and Department of Physics, Theory Division, CERN, 1211 Geneva 23, Switzerland
}

(Received 30 June 2006; published 8 September 2006)

\begin{abstract}
The tight coupling expansion, appropriately generalized to include large-scale magnetic fields, allows the estimate of the brightness perturbations of $\mathrm{CMB}$ anisotropies for typical wavelengths that are larger than the Hubble radius after matter-radiation equality. After discussing the basic features of the predecoupling initial conditions in the presence of fully inhomogeneous magnetic fields, the tight coupling expansion is studied both analytically and numerically. From the requirement that the amplitudes and phases of Sakharov oscillations are (predominantly) adiabatic and from the inferred value of the plateau in the temperature autocorrelation, the effects of the magnetized contribution can be systematically investigated and constrained.
\end{abstract}

DOI: 10.1103/PhysRevD.74.063002

PACS numbers: 98.70.Vc, 78.20.Ek, 98.62.En, 98.80.Cq

\section{INTRODUCTION}

A distinctive signature of predecoupling (adiabatic) initial conditions is that the temperature autocorrelation has the first (Doppler) peak for $\ell_{\mathrm{d}} \simeq 220$ [1-3]. For the same set of initial conditions, the cross-correlation power spectrum between temperature and polarization will have the first (anticorrelation) peak for $\ell_{\mathrm{c}} \simeq 3 \ell_{\mathrm{d}} / 4<\ell_{\mathrm{d}}$ [1].

Defining as $k$ the comoving wave number and as $c_{\mathrm{sb}}$ the sound speed of the baryon-photon system, the large-scale contribution to the temperature autocorrelation oscillates as a $\cos \left(k c_{\mathrm{sb}} \tau_{\text {dec }}\right)$, while the cross-correlation oscillates as $\sin \left(2 k c_{\mathrm{sb}} \tau_{\mathrm{dec}}\right)$. The first (compressional) peak of the temperature autocorrelation will then correspond to $k c_{\mathrm{sb}} \tau_{\mathrm{dec}} \sim$ $\pi$ (i.e. $\ell_{\mathrm{d}} \simeq 220$ ), while the first peak of the crosscorrelation will arise for $k c_{\mathrm{sb}} \tau_{\mathrm{dec}} \sim 3 \pi / 4$ (i.e., approximately $\ell_{\mathrm{c}} \simeq 3 \ell_{\mathrm{d}} / 4$ ). This result can be obtained analytically by working to first order in the tight coupling expansion [4-6]. In this framework, the key assumption is that the initial conditions of the Einstein-Boltzmann hierarchy are characterized, prior to matter-radiation equality, by a solution that is, at least predominantly, adiabatic. A corollary to the previous statements is that the peak in the cross-correlation occurs for typical wavelengths that are larger than the Hubble radius at the time of photon decoupling.

In a previous paper [7] it has been shown that large-scale magnetic fields, present prior to equality, affect the $\mathrm{CMB}$ initial conditions. If only the adiabatic mode is present, the fully inhomogeneous magnetic fields contribute to the Sachs-Wolfe (SW) plateau as a subdominant nonadiabatic component that may be either correlated or uncorrelated with the adiabatic mode. If the predecoupling initial conditions are given by a mixture of adiabatic and nonadiabatic modes, the magnetized contribution may also mix with the isocurvature modes so that the total number of

*Electronic address: massimo.giovannini@cern.ch parameters defining the initial conditions increases. It is desirable to complement and extend the analysis of [7] by scrutinizing the impact of predecoupling magnetic fields on intermediate scales. Within the tight coupling expansion, appropriately generalized to include the effects of fully inhomogeneous magnetic fields, the fluctuations in the brightness induced by the (adiabatic and nonadiabatic) scalar modes can be estimated.

The effects of magnetic fields on the scalar brightness perturbations are typically neglected. To give an example, let us consider how the impact of large-scale magnetic fields is usually computed, for instance, in the Faraday effect. This case is interesting, since the rotation of the CMB polarization plane indeed presupposes that the magnetic fields do not affect the process of formation of polarization. The influence of large-scale magnetic fields on CMB polarization can be separated, for illustration, into two physically distinct effects. Prior to decoupling, gravitating magnetic fields modify the initial conditions of $\mathrm{CMB}$ anisotropies and affect the evolution equations of the photon-baryon system. Therefore, the induced degree of polarization will be sensitive to the presence of magnetic fields whose role will be, at this stage, to modify the features of the adiabatic initial conditions. This is the first effect. Once the polarization is formed, the polarization plane of the CMB may be Faraday rotated [8,9]. From this second effect, interesting bounds on the magnetic field intensity are usually derived.

This philosophy motivated various independent studies on the possible Faraday rotation of the CMB polarization degree [10-13]. In some cases the magnetic field has been assumed just uniform [10], but there are also calculations analyzing the situation where the magnetic field is fully inhomogeneous $[11,12]$. In spite of the relevant technical differences, the common assumption of the mentioned investigations is that the magnetic field is negligible in the process of formation of $\mathrm{CMB}$ polarization. The latter assumption can be rephrased by saying that the initial 
conditions of the Einstein-Boltzmann hierarchy are set in such a way that the magnetic fields are absent. In the absence of large-scale magnetic fields, the only source of polarization arises to first order in the tight coupling expansion. The magnitude of the obtained contribution is just a consequence of the adiabaticity of the solution. If largescale magnetic fields are consistently introduced before matter-radiation equality, the usual adiabatic (as well as nonadiabatic) initial conditions of the Einstein-Boltzmann hierarchy are modified and, consequently, the degree of polarization of the CMB is also affected.

This example motivates a systematic analysis of the impact of magnetized initial conditions on the brightness perturbations of the radiation field. As anticipated above, this calculation will be conducted within the tight coupling approximation which is known to be rather effective in obtaining the brightness perturbations for large and intermediate scales. This analysis has never been done before, to the best of our knowledge. A related motivation is that, in the absence of magnetic fields, the tight coupling approximation is employed in numerical Boltzmann codes at early times to avoid the integration of stiff Euler equations. The results of the present investigation allow us to use the same strategy also in the presence of fully inhomogeneous magnetic fields.

The example of Faraday rotation used in the previous paragraphs to highlight the relevance of the approach of the present paper should not give the wrong impression that previous works have only concentrated on the Faraday rotation effects due to large-scale magnetic fields. Indeed, various investigations treated different aspects of the CMB anisotropies (as well as the polarization power spectra) induced by vector and tensor modes. These works will be summarized in the following paragraphs. In spite of this, the scalar modes induced by CMB anisotropies have been comparatively less studied, as we shall see in a moment.

Several aspects of CMB physics are germane to largescale magnetization (see [14,15] for two topical review articles). The astrophysical evidence for large-scale magnetization can be summarized as follows. Large-scale magnetic fields are an important element of the physics of the interstellar medium and have been measured, through various techniques, in galaxies [16,17] and in rich clusters [18-21]. The determination of large-scale magnetic fields associated with loosely gravitationally bound systems (like superclusters) is still debatable [2224] but, nonetheless, extremely intriguing. As far as galactic magnetic fields are concerned, there is the hope that in the future all sky survey of Faraday rotation [achievable through the square kilometer array (SKA) [17,18]] will allow a Faraday "tomography" of the Milky Way, addressing the dark corners (number of reversals, pitch angle) of the morphological structure of the closest large-scale magnetic field we observe through different surveys of pulsars such as the Parkes and the southern galactic plane surveys (see [25-27] and references therein). There is the hope, in the present framework, that the present-day coherent field in spiral galaxies may keep the "memory" of the structure of the initial seed field (see [16] for a review of this longstanding problem). The morphological features of the magnetic field profiles in clusters are less understood than in the case of galaxies. However, attempts have been made in constraining the radial profiles of magnetic fields in clusters $[19,20]$. Determinations of intracluster magnetic fields through statistical samples of rich clusters [21] seem to suggest that (ordered?) intracluster magnetic fields may be as large as the $\mu \mathrm{G}$ (see, for instance, [28] for a review on the features of our magnetized Universe).

Compressional amplification (taking place during the gravitational collapse of the protogalaxy) allows us to connect the observed field to a protogalactic field, present prior to gravitational collapse, between $0.1 \mathrm{nG}$ and $0.01 \mathrm{nG}$. It is plausible, within the dynamo hypothesis, that the protogalactic fields could even be much smaller than the nG. In recent years, a lot of progress has been made, in the context of both small- and large-scale dynamos i.e. the process by which the kinetic energy of the protogalactic plasma is transferred, by differential rotation, into magnetic energy. This progress [29] (see also [30] for an introduction of astrophysical dynamos) has been driven both by the higher resolution of numerical simulations and by the improvement in the understanding of the largest magnetized system that is close to us, i.e. the sun. In contrast with the previous lore, it is now clear that the dynamo action demands a change in the topology of the flux lines. As a consequence, large-scale dynamos should also produce small-scale helical fields that quench (i.e. prematurely saturate) the $\alpha$ effect [29] (see also [31]). The morphology and strength of magnetic fields in clusters may be related to the way the dynamo effect saturates.

The simplest way to understand why large-scale magnetic fields may be relevant for CMB physics is to think of a protogalactic magnetic field in the $\mathrm{nG}$ range. If this field is naively blue-shifted at the decoupling epoch, i.e. at a redshift $z_{\mathrm{dec}} \simeq 1100$, its strength could be as large as the $\mathrm{mG}$, i.e. roughly 6 orders of magnitude larger. In the past ten years, indeed a lot of work has been done with the purpose of constraining large-scale magnetic fields using CMB physics. Historically, the first types of configurations used in this type of exercise have been weakly uniform magnetic fields that would partially break the isotropy of the background geometry [15]. It was than realized that this assumption induces a correlation of the $a_{\ell-1, m}$ and $a_{\ell+1, m}$ multipole coefficients arising in the angular power spectrum (see [32-34] and references therein). From this observation, uniform magnetic fields can be constrained.

Another (complementary) possibility is that magnetic fields are fully inhomogeneous. In this case the magnetic fields do not break the spatial isotropy of the background 
but they can affect virtually all observables relevant in the theory of CMB anisotropies. In particular, in a series of papers, Barrow and Subramanian [35-37] pointed out possible effects of magnetic fields on the induced vector CMB anisotropies as well as on the polarization power spectra [38] induced by the same modes. Within a slightly different approach the vector modes (as well as the tensor modes) have been discussed in [39].

As previously stressed, the attention paid in the previous paragraphs to the Faraday effect should not be misunderstood. In the standard scenario of inflationary generated perturbations, we do know that polarization is generated at last scattering. If magnetic fields are present, the induced vector modes also affect the polarization power spectra as clearly discussed, for instance, in [38-41]. While the polarization power spectra induced by the scalar modes depend simultaneously on the nature of the initial adiabatic (or nonadiabatic) solution and on the magnetic field properties, the polarization patterns induced by the vector modes only depend upon the features of the magnetic fields since vector modes are absent in the standard paradigm of structure formation.

More recently [40] a full numerical analysis of the vector and of the tensor modes induced by fully inhomogeneous magnetic fields has been presented. In [41] the signatures of magnetic helicity have been studied, always in the perspective of the vector modes. Finally, in a series of papers, the evolution of weakly inhomogeneous magnetic fields has been discussed within the covariant formalism and in the presence of a uniform magnetic background [42].

In spite of the fact that vector modes are probably one of the characteristic signatures of large-scale magnetic fields, scalar modes are also induced $[7,43]$. This statement can be understood by noticing that the magnetic energy density, the magnetic anisotropic stress and the magnetic pressure contribute, respectively, to the Hamiltonian and momentum constraint and to the dynamical evolution of the curvature perturbations. Furthermore, within the magnetohydrodynamical approximation, the divergence of the Lorentz force contributes to the evolution equation of the divergence-full part of the baryon peculiar velocity.

What is missing, at the moment, is the analysis of the brightness perturbations induced by magnetized initial conditions. As anticipated, one of the purposes of the present paper will exactly be to fill this gap and to allow an approximate evaluation of the brightness perturbations, also at intermediate scales, as a function of the spectral properties of fully inhomogeneous magnetic fields.

Before closing the present introduction, it is useful to stress the limitations of the present analysis. In order to assess quantitatively the relevance of a given parameter for CMB anisotropies, one may take two different approaches. According to the first strategy, the whole set of parameters determining the properties of scalar CMB anisotropies has to be analyzed with statistical methods. The purpose of the present paper is, however, more modest; we would like to analyze the effect of large-scale magnetic fields in the tight coupling approximation when the initial conditions of the system consistently include large-scale magnetic fields. This second approach is not only interesting per se but it also represents a necessary preliminary step for more complete statistical analyses. Since such a discussion is missing in the present treatments of magnetized scalar perturbations, it is appropriate to concentrate on the second approach discussed above and to leave the possible strategies of parameter extraction for future investigations.

The present paper is then organized as follows. In Sec. II the initial conditions of the Einstein-Boltzmann hierarchy will be discussed for typical wavelengths larger than the Hubble radius prior to equality. In Sec. III the tight coupling equations will be derived and discussed analytically. The numerical treatment of the system is presented in Sec. IV. Section V contains our concluding remarks.

\section{ADIABATIC AND NONADIABATIC MODES}

\section{A. Zeroth-order tight coupling approximation}

By combining the Thompson cross section (defined, in the following, as $\sigma_{\mathrm{T}}$ ) with the ionization fraction $x_{\mathrm{e}}$ and with the electron density $n_{\mathrm{e}}$, the differential optical depth is given by $\kappa^{\prime}=n_{\mathrm{e}} x_{\mathrm{e}} \sigma_{\mathrm{T}} a / a_{0}$. Since prior to decoupling the photon mean free path is much smaller than the Hubble radius at the corresponding epoch, the inverse of $\kappa^{\prime}$ can be used as an expansion parameter. This is the basic idea of the tight coupling approximation. To lowest order, the tight coupling approximation implies $1 / \kappa^{\prime} \rightarrow 0$ (or, equivalently, that $\left.\sigma_{\mathrm{T}} \rightarrow \infty\right)$. In this limit the divergences of the peculiar velocities of photons (i.e. $\theta_{\gamma}$ ) and baryons (i.e. $\theta_{\mathrm{b}}$ ) are driven to a common value, i.e. $\theta_{\gamma} \simeq \theta_{\mathrm{b}}=\theta_{\gamma \mathrm{b}}$. In the following, the consequences of the zeroth order in the tight coupling approximation will be investigated in the presence of fully inhomogeneous magnetic fields. Consider, therefore, the case where large-scale magnetic fields are fully inhomogeneous and characterized, in Fourier space, by the following two-point function:

$$
\left\langle B_{i}(\vec{k}, \tau) B^{j}(\vec{p}, \tau)\right\rangle=\frac{2 \pi^{2}}{k^{3}} P_{i}^{j}(k) P_{\mathrm{B}}(k) \delta^{(3)}(\vec{k}+\vec{p}),
$$

where

$$
P_{i}^{j}(k)=\left(\delta_{i}^{j}-\frac{k_{i} k^{j}}{k^{2}}\right), \quad P_{\mathrm{B}}(k)=A_{\mathrm{B}}\left(\frac{k}{k_{\mathrm{p}}}\right)^{\varepsilon} .
$$

In Eq. (2.2) $k_{\mathrm{p}}$ is the "pivot" scale at which the spectra are normalized [see also Eq. (2.35)]. Since the magnetic fields are fully inhomogeneous, the line element of the back- 
ground geometry will not be anisotropic ${ }^{1}$ (as it happens, for instance, in magnetized Bianchi-type backgrounds) and it can be parametrized as

$$
d s^{2}=a^{2}(\tau)\left[d \tau^{2}-d \vec{x}^{2}\right]
$$

Consequently, the evolution equations of the background geometry will be solely determined by the total pressure and energy density of the system as

$$
\begin{aligned}
\mathcal{H}^{2}=\frac{8 \pi G}{3} a^{2} \rho_{\mathrm{t}}, \quad \mathcal{H}^{2}-\mathcal{H}^{\prime} & =4 \pi G a^{2}\left(\rho_{\mathrm{t}}+p_{\mathrm{t}}\right) \\
\rho_{\mathrm{t}}^{\prime}+3 \mathcal{H}\left(\rho_{\mathrm{t}}+p_{\mathrm{t}}\right) & =0
\end{aligned}
$$

where $\mathcal{H}=a^{\prime} / a$ and the prime denotes a derivation with respect to the conformal time coordinate $\tau$. Denoting the longitudinal fluctuations of the metric (2.3) as ${ }^{2}$

$$
\delta_{\mathrm{s}} g_{00}=2 a^{2} \phi, \quad \delta_{\mathrm{s}} g_{i j}=2 a^{2} \psi \delta_{i j}
$$

and selecting the conformally Newtonian gauge [46,47], the evolution equations of photons and baryons can be obtained in terms of the density contrasts (i.e. $\delta_{\gamma}$ and $\delta_{\mathrm{b}}$ ) and in terms of the divergence of the peculiar velocities (i.e. $\theta_{\gamma}$ and $\theta_{\mathrm{b}}$ ):

$$
\begin{gathered}
\delta_{\gamma}^{\prime}=4 \psi^{\prime}-\frac{4}{3} \theta_{\gamma}, \\
\theta_{\gamma}^{\prime}=-\frac{1}{4} \nabla^{2} \delta_{\gamma}-\nabla^{2} \phi+\kappa^{\prime}\left(\theta_{\mathrm{b}}-\theta_{\gamma}\right), \\
\delta_{\mathrm{b}}^{\prime}=3 \psi^{\prime}-\theta_{\mathrm{b}}, \\
\theta_{\mathrm{b}}^{\prime}=-\mathcal{H} \theta_{\mathrm{b}}-\nabla^{2} \phi+\frac{\vec{\nabla} \cdot[\vec{J} \times \vec{B}]}{a^{4} \rho_{\mathrm{b}}}+\frac{4}{3} \frac{\rho_{\gamma}}{\rho_{\mathrm{b}}} \kappa^{\prime}\left(\theta_{\gamma}-\theta_{\mathrm{b}}\right) .
\end{gathered}
$$

The term $\vec{J} \times \vec{B}$ parametrizes the Lorentz force contribution as it arises in a conducting plasma which is, however, globally neutral (see, for instance, [48,49]). In this situation, typical of magnetohydrodynamics (MHD), the possible contribution of electric fields is suppressed by inverse powers of the conductivity, as it will be discussed later. By subtracting the two equations for the velocities introduced in (2.6) and (2.7), the combination $\left(\theta_{\gamma}-\theta_{\mathrm{b}}\right)$ obeys

\footnotetext{
${ }^{1}$ If the expansion is anisotropic because of a uniform magnetic field oriented along a specific direction, as in the celebrated Zeldovich models $[44,45]$, the angular power spectrum does not depend solely upon $\ell$ but also upon $m$. These situations can be successfully constrained through WMAP data (see [32-34]) and references therein.

${ }^{2}$ In Eq. (2.5) and in what follows, $\delta_{\mathrm{s}}(\ldots)$ denotes the scalar fluctuation of the corresponding quantity.
}

$$
\begin{gathered}
\left(\theta_{\gamma}-\theta_{\mathrm{b}}\right)^{\prime}+\kappa^{\prime}\left(1+\frac{4}{3} \frac{\rho_{\gamma}}{\rho_{\mathrm{b}}}\right)\left(\theta_{\gamma}-\theta_{\mathrm{b}}\right) \\
=-\frac{1}{4} \nabla^{2} \delta_{\gamma}+\mathcal{H} \theta_{\mathrm{b}}+\frac{\vec{\nabla} \cdot[\vec{J} \times \vec{B}]}{a^{4} \rho_{\mathrm{b}}} .
\end{gathered}
$$

The term on the right-hand side of Eq. (2.8) can be viewed as a source term for the evolution of the difference between the divergences of the photon and baryon peculiar velocities. In spite of the magnitude of the source term on the right-hand side of Eq. (2.8), $\left(\theta_{\gamma}-\theta_{\mathrm{b}}\right)$ will quickly be driven to zero in the limit $\sigma_{\mathrm{T}} \rightarrow \infty$. Consequently, even if predecoupling magnetic fields are present, the lowest order tight coupling expansion still implies that $\theta_{\gamma} \rightarrow \theta_{\mathrm{b}}$ as expected.

In the tightly coupled limit, the evolution equation of $\theta_{\gamma \mathrm{b}}=\theta_{\gamma}=\theta_{\mathrm{b}}$ can then be swiftly obtained by summing up the equations for the velocity fields in such a way that the scattering terms cancel. The result of this procedure is, in Fourier space,

$$
\begin{aligned}
\theta_{\gamma \mathrm{b}}^{\prime}+\frac{\mathcal{H} R_{\mathrm{b}}}{R_{\mathrm{b}}+1} \theta_{\gamma \mathrm{b}}= & \frac{k^{2}}{4\left(1+R_{\mathrm{b}}\right)} \delta_{\gamma}+k^{2} \phi \\
& +\frac{k^{2}}{4\left(1+R_{\mathrm{b}}\right)}\left(\Omega_{\mathrm{B}}-4 \sigma_{\mathrm{B}}\right),
\end{aligned}
$$

where $R_{\mathrm{b}}=(3 / 4) \rho_{\mathrm{b}} / \rho_{\gamma}$. For numerical purposes (see Sec. IV) the late-time cosmological parameters will be fixed, for a spatially flat universe, as ${ }^{3}$

$$
\begin{gathered}
\omega_{\gamma}=2.47 \times 10^{-5}, \quad \omega_{\mathrm{b}}=0.023, \\
\omega_{\mathrm{c}}=0.111, \quad \omega_{\mathrm{m}}=\omega_{\mathrm{b}}+\omega_{\mathrm{c}},
\end{gathered}
$$

where $\omega_{X}=h^{2} \Omega_{X}$ and $\Omega_{\Lambda}=1-\Omega_{\mathrm{m}}$; the present value of the Hubble parameter $H_{0}$ will be fixed, for numerical estimates, to 73 in units of $\mathrm{km} /(\mathrm{sec} \mathrm{Mpc})$. In terms of the fiducial set of parameters of Eq. (2.10), the baryon to photon ratio appearing in Eq. (2.9) becomes

$$
R_{\mathrm{b}}=\left(\frac{698}{z+1}\right)\left(\frac{\omega_{\mathrm{b}}}{0.023}\right)\left(\frac{\omega_{\gamma}}{2.47 \times 10^{-5}}\right)^{-1}
$$

where $z+1=a_{0} / a$ is the redshift [for numerical purposes the scale factor will be normalized in such a way that $a_{0}=1$; see below, Eq. (2.25)]. In Eq. (2.9) we traded the MHD current $\vec{J}$ [appearing in the second equation of

\footnotetext{
${ }^{3}$ The values of the cosmological parameters introduced in Eq. (2.10) are compatible with the ones estimated from WMAP-3 [2,3] in combination with the "Gold" sample of SNIa [50] consisting of 157 supernovae (the furthest being at redshift $z=1.75)$. We are aware of the fact that WMAP-3 data alone seem to favor a slightly smaller value of $\omega_{\mathrm{m}}$ (i.e. 0.126). Moreover, WMAP-3 data may also have slightly different implications if combined with supernovae of the SNLS project [51]. The values given in Eq. (2.10) will just be used for a realistic numerical illustration of the methods developed in the present investigation.
} 
(2.7)] for a combination of the magnetic energy density and of the anisotropic stress. More specifically, using the results reported in Eqs. (A1) and (A2) of the Appendix, it can be easily shown that

$$
\begin{gathered}
\frac{3}{4} \frac{\vec{\nabla} \cdot[\vec{J} \times \vec{B}]}{a^{4} \rho_{\gamma}}=\nabla^{2} \sigma_{\mathrm{B}}-\frac{1}{4} \nabla^{2} \Omega_{\mathrm{B}}, \quad \Omega_{\mathrm{B}}=\frac{\delta \rho_{\mathrm{B}}}{\rho_{\gamma}}, \\
\delta \rho_{\mathrm{B}}=\frac{B^{2}}{8 \pi a^{4}} .
\end{gathered}
$$

Equation (2.12) holds since, in MHD, the total Ohmic current $\vec{J}$ is solenoidal (i.e. $\vec{\nabla} \cdot \vec{J}=0$ ) and given by $4 \pi \vec{J}=$ $\vec{\nabla} \times \vec{B}[48,49]$ (see also the Appendix). In addition, the function $\sigma_{\mathrm{B}}$ is nothing but a parametrization of the anisotropic stress that appears in the spatial part of the magnetic energy-momentum tensor, i.e.

$$
\delta_{\mathrm{s}} \mathcal{T}_{i}^{j}=-\delta p_{\mathrm{B}}+\tilde{\Pi}_{i}^{j}, \quad \partial_{i} \partial^{j} \tilde{\Pi}_{i}^{j}=\left(p_{\gamma}+\rho_{\gamma}\right) \nabla^{2} \sigma_{\mathrm{B}},
$$

where

$$
\delta p_{\mathrm{B}}=\frac{\delta \rho_{\mathrm{B}}}{3}, \quad \tilde{\Pi}_{i}^{j}=\frac{1}{4 \pi a^{4}}\left(B_{i} B^{j}-\frac{B^{2}}{3} \delta_{i}^{j}\right) .
$$

In various contexts, the force-free approximation is often employed [52,53] (see also [28] and references therein). Such an approximation amounts, in practice, to setting $(\vec{\nabla} \times \vec{B}) \times \vec{B} \rightarrow 0$. In this limit, from Eq. (2.12) $\sigma_{\mathrm{B}} \rightarrow$ $\Omega_{\mathrm{B}} / 4$. In the present framework this assumption shall not be invoked.

According to Eq. (2.12), the leading contribution of fully inhomogeneous magnetic fields to the scalar modes of the geometry is given by the magnetic energy density and by the divergence of the MHD Lorentz force. These two quantities affect the perturbed Einstein equations and, in particular, the Hamiltonian constraint

$$
\nabla^{2} \psi-3 \mathcal{H}\left(\mathcal{H} \phi+\psi^{\prime}\right)=4 \pi G a^{2}\left[\delta \rho_{\mathrm{t}}+\delta_{\mathrm{s}} \rho_{\mathrm{B}}\right],
$$

and the total (scalar) anisotropic stress

$$
\begin{aligned}
\nabla^{4}(\phi-\psi)= & 12 \pi G a^{2}\left[\left(p_{\nu}+\rho_{\nu}\right) \nabla^{2} \sigma_{\nu}\right. \\
& \left.+\left(p_{\gamma}+\rho_{\gamma}\right) \nabla^{2} \sigma_{\mathrm{B}}\right] .
\end{aligned}
$$

Equations (2.15) and (2.16) follow, respectively, from the (00) and $(i \neq j)$ components of the perturbed Einstein equations written in the conformally Newtonian coordinate system; the quantity $\delta_{\mathrm{s}} \rho_{\mathrm{t}}$ is the (total) scalar fluctuation of the energy density of the fluid sources. It is relevant to remark that, in Eq. (2.16), on top of the anisotropic stress of the magnetic field, the only source of the anisotropic stress of the fluid is provided by the neutrinos that are collisionless after weak interactions fall out of thermal equilibrium for typical temperatures of the order of $0.8 \mathrm{MeV}$. In analogy with Eq. (2.13), the anisotropic stress of the neutrinos has been parametrized as

$$
\delta_{\mathrm{s}} T_{i}^{j}=-\delta_{\mathrm{s}} p_{\mathrm{t}}+\Pi_{i}^{j}, \quad \partial_{i} \partial^{j} \Pi_{i}^{j}=\left(p_{\nu}+\rho_{\nu}\right) \nabla^{2} \sigma_{\nu} .
$$

The quantity $\delta_{\mathrm{s}} p_{\mathrm{t}}$ is the fluctuation of the total pressure. The last constraint on the dynamical evolution is derived from the $(0 i)$ components of the Einstein equations and it is

$$
\nabla^{2}\left(\mathcal{H} \phi+\psi^{\prime}\right)=-4 \pi G a^{2}\left[\left(p_{\mathrm{t}}+\rho_{\mathrm{t}}\right) \theta_{\mathrm{t}}+\frac{\vec{\nabla} \cdot(\vec{E} \times \vec{B})}{4 \pi a^{4}}\right] .
$$

The second term on the right-hand side of Eq. (2.18) is the divergence of the Poynting vector. In MHD the Ohmic electric field is subleading and, in particular, from the MHD expression of the Ohm law,

$$
\vec{E} \times \vec{B}=\frac{(\vec{\nabla} \times \vec{B}) \times \vec{B}}{4 \pi \sigma} .
$$

Since the Universe, prior to decoupling, is a very good conductor, the ideal MHD limit [49] can be safely adopted in the first approximation (see also [54]); thus, for $\sigma \rightarrow \infty$ the contribution of the Poynting vector vanishes. In any case, even if $\sigma$ is finite but large, the second term is suppressed in comparison with the first term on the right-hand side of Eq. (2.18); such a term is given by the sum of the divergence of the peculiar velocity of each fluid component weighted by the specific enthalpy [i.e. $\left(p_{\mathrm{a}}+\rho_{\mathrm{a}}\right)$ for a generic a-fluid]:

$$
\left(p_{\mathrm{t}}+\rho_{\mathrm{t}}\right) \theta_{\mathrm{t}}=\sum_{\mathrm{a}}\left(p_{\mathrm{a}}+\rho_{\mathrm{a}}\right) \theta_{\mathrm{a}},
$$

or, in more explicit terms,

$$
\begin{aligned}
\left(p_{\mathrm{t}}+\rho_{\mathrm{t}}\right) \theta_{\mathrm{t}} & \equiv \frac{4}{3} \rho_{\nu} \theta_{\nu}+\frac{4}{3} \rho_{\gamma} \theta_{\gamma}+\rho_{\mathrm{c}} \theta_{\mathrm{c}}+\rho_{\mathrm{b}} \theta_{\mathrm{b}} \\
& =\frac{4}{3} \rho_{\nu} \theta_{\nu}+\rho_{\mathrm{c}}+\frac{4}{3} \rho_{\gamma}\left(1+R_{\mathrm{b}}\right) \theta_{\gamma \mathrm{b}} .
\end{aligned}
$$

The second equality in Eq. (2.21) follows from the zeroth order in the tight coupling expansion and from the definition of the baryon to photon ratio (2.11). Every regular solution to lowest order in the tight coupling expansion has to satisfy the momentum constraint of Eq. (2.18). Such a requirement implies, in particular, a restriction on the form of the peculiar velocity fields for comoving wave numbers larger than the Hubble radius (i.e. $k \tau \ll 1$ ).

The evolution of the cold dark matter (CDM) component only feels the presence of the magnetic field through the modifications induced by $\Omega_{\mathrm{B}}$ and $\sigma_{\mathrm{B}}$ on the two conformally Newtonian potentials, i.e. $\phi$ and $\psi$ :

$$
\delta_{\mathrm{c}}^{\prime}=3 \psi^{\prime}-\theta_{\mathrm{c}}, \quad \theta_{\mathrm{c}}^{\prime}+\mathcal{H} \theta_{\mathrm{c}}=k^{2} \phi .
$$

A similar situation occurs for the evolution of the neutrino component where, however, the effect of the magnetic field enters not only the Newtonian potentials but also the anisotropic stress that is constrained from Eq. (2.16). The relevant equations for the neutrinos are then, in Fourier space, 


$$
\begin{gathered}
\delta_{\nu}^{\prime}=4 \psi^{\prime}-\frac{4}{3} \theta_{\nu}, \quad \theta_{\nu}^{\prime}=\frac{k^{2}}{4} \delta_{\nu}-k^{2} \sigma_{\nu}+k^{2} \phi, \\
\sigma_{\nu}^{\prime}=\frac{4}{15} \theta_{\nu}-\frac{3}{10} k \mathcal{F}_{\nu 3} .
\end{gathered}
$$

The quantity $\mathcal{F}_{\nu 3}$ appearing in Eq. (2.23) is the octupole of the (perturbed) neutrino phase-space distribution while $\sigma_{\nu}$ [i.e. the anisotropic stress of the neutrinos introduced in Eqs. (2.16) and (2.17)] is related to the quadrupole moment of $\mathcal{F}_{\nu}$ as $\sigma_{\nu}=\mathcal{F}_{\nu 2} / 2$. The rationale for the inclusion of $\mathcal{F}_{\nu 3}$ in the fluid description is that neutrinos are collisionless for temperatures smaller than $0.8 \mathrm{MeV}$ when the Universe is still deep into the radiation-dominated regime. In this regime the neutrinos do not interact, and, consequently, they should rather be described by the collisionless Boltzmann hierarchy of the (perturbed) neutrino phase-space distribution. To address this problem, the usual approach is to use an improved description where, on top of the monopole and dipole of the (perturbed) neutrino phase-space distribution (connected, respectively, with $\delta_{\nu}$ and $\theta_{\nu}$ ), one also takes into account the quadrupole, i.e. $\sigma_{\nu}$, and sometimes (as in the case of specific nonadiabatic modes) also the octupole.

With all these ingredients in mind, the evolution of the longitudinal fluctuations of the geometry is determined from the trace spatial components of the perturbed Einstein equations, and can be written as

$$
\begin{aligned}
\psi^{\prime \prime} & +\mathcal{H}\left(\phi^{\prime}+2 \psi^{\prime}\right)+\left(\mathcal{H}^{2}+2 \mathcal{H}^{\prime}\right) \phi+\frac{1}{3} \nabla^{2}(\phi-\psi) \\
& =4 \pi G a^{2}\left(\delta_{\mathrm{s}} p_{\mathrm{t}}+\delta p_{\mathrm{B}}\right),
\end{aligned}
$$

where $\delta_{\mathrm{s}} p_{\mathrm{t}}$ is the fluctuation of the total pressure introduced in Eq. (2.17). In the present situation, it will be mandatory to set initial conditions for the fluctuations of the geometry and for the various species of the plasma before radiation-matter equality but, also, after neutrino decoupling. In the conformally flat case described by Eq. (2.3), the scale factor that interpolates between the radiation epoch and the matter-dominated epoch can be obtained by integrating Eqs. (2.4):

$$
\begin{aligned}
a(\tau) & =a_{\mathrm{eq}}\left[\left(\frac{\tau}{\tau_{1}}\right)^{2}+2\left(\frac{\tau}{\tau_{1}}\right)\right], \\
1+z_{\mathrm{eq}} & =\frac{1}{a_{\mathrm{eq}}}=\frac{h^{2} \Omega_{\mathrm{m} 0}}{h^{2} \Omega_{\mathrm{r} 0}},
\end{aligned}
$$

where $\Omega_{\mathrm{m} 0}$ and $\Omega_{\mathrm{r} 0}$ are evaluated at the present time and the scale factor is normalized in such a way that $a_{0}=1$. In Eq. (2.25), $\tau_{1}=\left(2 / H_{0}\right) \sqrt{a_{\mathrm{eq}} / \Omega_{\mathrm{m} 0}}$. In terms of $\tau_{1}$ the equality time is

$$
\tau_{\mathrm{eq}}=(\sqrt{2}-1) \tau_{1}=119.07\left(\frac{h^{2} \Omega_{\mathrm{m} 0}}{0.134}\right)^{-1} \mathrm{Mpc},
$$

i.e. $2 \tau_{\text {eq }} \simeq \tau_{1}$. In this framework the total optical depth from the present to the critical recombination epoch, i.e. $800<z<1200$, can be approximated analytically, as discussed in [55]. By defining the redshift of decoupling as the one where the total optical depth is of order 1, i.e. $\kappa\left(z_{\mathrm{dec}}, 0\right) \simeq 1$, we will have, approximately,

$$
\begin{aligned}
z_{\mathrm{dec}} & \simeq 1139\left(\frac{\Omega_{\mathrm{b}}}{0.0431}\right)^{-\alpha_{1}}, \\
\alpha_{1} & =\frac{0.0268}{0.6462+0.1125 \ln \left(\Omega_{\mathrm{b}} / 0.0431\right)},
\end{aligned}
$$

where, consistently with Eq. (2.10), $h=0.73$. From Eqs. (2.25) and (2.27) it follows that for $1100 \leq z_{\mathrm{dec}} \leq$ $1139,275 \mathrm{Mpc} \leq \tau_{\mathrm{dec}} \leq 285 \mathrm{Mpc}$.

\section{B. Magnetized adiabatic mode}

In the limit $\tau / \tau_{1} \ll 1$, the solution of the whole system of governing equations corresponding to the adiabatic mode can be obtained as an expansion in powers of $k \tau$. When the given wavelength is larger than the Hubble radius before equality (i.e. $k \tau<1$ for $\tau<\tau_{\text {eq }}$ ), the solution of the coupled system introduced in the previous subsection can be written as ${ }^{4}$

$$
\begin{gathered}
\delta_{\nu}=\delta_{\gamma}=-2 \phi_{*}-R_{\gamma} \Omega_{\mathrm{B}} \\
\delta_{\mathrm{c}}=\delta_{\mathrm{b}}=-\frac{3}{2} \phi_{*}-\frac{3}{4} R_{\gamma} \Omega_{\mathrm{B}} \\
\theta_{\mathrm{c}}=\frac{k^{2} \tau}{2} \phi_{*}, \quad \theta_{\nu}=\frac{k^{2} \tau}{2}\left[\phi_{*}-\frac{R_{\gamma} \Omega_{\mathrm{B}}}{2}+2 \frac{R_{\gamma}}{R_{\nu}} \sigma_{\mathrm{B}}\right] \\
\theta_{\gamma \mathrm{b}}=\frac{k^{2} \tau}{2}\left[\phi_{*}+\frac{R_{\nu} \Omega_{\mathrm{B}}}{2}-2 \sigma_{\mathrm{B}}\right] \\
\psi_{*}=\phi_{*}\left(1+\frac{2}{5} R_{\nu}\right)+\frac{R_{\gamma}}{5}\left[4 \sigma_{\mathrm{B}}-R_{\nu} \Omega_{\mathrm{B}}\right] \\
\sigma_{\nu}=-\frac{R_{\gamma}}{R_{\nu}} \sigma_{\nu}+\frac{k^{2} \tau^{2}}{6 R_{\nu}}\left(\psi_{*}-\phi_{*}\right),
\end{gathered}
$$

where the fractional contribution of photons to the radiation plasma, i.e. $R_{\gamma}$, has been introduced and it is related to $R_{\nu}$, i.e. the fractional contribution of massless neutrinos, as

\footnotetext{
${ }^{4}$ In the following, all the perturbed quantities will be defined in Fourier space and will depend on the comoving wave number $k$. ${ }^{5}$ In the present paper $h^{2} \Omega_{\nu}=1.68 \times 10^{-5}$, i.e., recalling Eq. (2.10), $h^{2} \Omega_{\mathrm{r} 0}=h^{2} \Omega_{\gamma}+h^{2} \Omega_{\nu}=4.15 \times 10^{-5}$. According to Eq. (2.25) this implies that $1+z_{\mathrm{eq}} \simeq 3228.9\left(\omega_{\mathrm{m}} / 0.134\right)$. Possible mass terms for the neutrinos in the $\mathrm{MeV}$ range will be neglected here but can be introduced with a modification of the neutrino Boltzmann hierarchy used to derive the relations reported in Eq. (2.23).
} 


$$
\begin{aligned}
& R_{\gamma}=1-R_{\nu}, \quad R_{\nu}=\frac{r}{1+r}, \\
& r=\frac{7}{8} N_{\nu}\left(\frac{4}{11}\right)^{4 / 3} \equiv 0.681\left(\frac{N_{\nu}}{3}\right) .
\end{aligned}
$$

In Eq. (2.31) $\phi_{*}(k)$ and $\psi_{*}(k)$ denote the values of the Fourier modes of the longitudinal fluctuations of the geometry that are (approximately) constant in time. Equations (2.28), (2.29), and (2.31) define a solution that is adiabatic since the density contrasts in matter (i.e. $\delta_{\mathrm{c}}$ and $\delta_{\mathrm{c}}$ ) are exactly $3 / 4$ of the density contrasts in radiation (i.e. $\delta_{\gamma}$ and $\delta_{\nu}$ ). In the limit $\Omega_{\mathrm{B}} \rightarrow 0$ (vanishing magnetic energy density) and $\sigma_{\mathrm{B}} \rightarrow 0$ (vanishing magnetic anisotropic stress), Eqs. (2.28), (2.29), and (2.31) reproduce the standard adiabatic mode deep in the radiation-dominated epoch [43,56]. From the second equation reported in (2.31), it is clear that the neutrino anisotropic stress is partially counterbalanced by the magnetic anisotropic stress. This aspect is entirely due to the presence of the magnetic anisotropic stress and it has been verified in different situations also involving entropic fluctuations [57].

In view of the forthcoming numerical applications, it is useful to characterize the adiabatic mode in fully gaugeinvariant terms. In fact, the quantity introduced so far is meaningful in the longitudinal gauge. The spectrum of curvature perturbations used as an initial condition for the numerical evaluation of the brightness perturbations is often introduced [1-3] in terms of the curvature perturbations on comoving orthogonal hypersurfaces [58,59], customarily denoted with $\mathcal{R}$. In the longitudinal gauge we have that this quantity is simply given by

$$
\mathcal{R}=-\psi-\frac{\mathcal{H}\left(\mathcal{H} \phi+\psi^{\prime}\right)}{\mathcal{H}^{2}-\mathcal{H}^{\prime}}
$$

In spite of the fact that $\mathcal{R}$ is here defined in terms of the variables of the longitudinal gauge, its value is invariant under infinitesimal coordinate transformations. In terms of $\mathcal{R}$ the longitudinal fluctuations of the geometry entering the solution of Eqs. (2.28), (2.29), and (2.31) become

$$
\begin{aligned}
& \phi_{*}=-\frac{10 \mathcal{R}_{*}}{15+4 R_{\nu}}-\frac{2 R_{\gamma}\left(4 \sigma_{\mathrm{B}}-R_{\nu} \Omega_{\mathrm{B}}\right)}{\left(15+4 R_{\nu}\right)}, \\
& \psi_{*}=-\frac{2\left(5+2 R_{\nu}\right)}{15+4 R_{\nu}} \mathcal{R}_{*}+\frac{R_{\gamma}}{15+4 R_{\nu}}\left[4 \sigma_{\mathrm{B}}-R_{\nu} \Omega_{\mathrm{B}}\right],
\end{aligned}
$$

where $\mathcal{R}_{*}(k)$ is the constant value of curvature perturbations prior to matter-radiation equality. Within the notations followed in this paper, the spectrum of $\mathcal{R}_{*}(k)$ is defined as

$$
\begin{aligned}
\left\langle\mathcal{R}_{*}(\vec{k}) \mathcal{R}_{*}(\vec{p})\right\rangle & =\frac{2 \pi^{2}}{k^{3}} \mathcal{P}_{\mathcal{R}}(k) \delta^{(3)}(\vec{k}+\vec{p}), \\
\mathcal{P}_{\mathcal{R}}(k) & =A_{\mathcal{R}}\left(\frac{k}{k_{\mathrm{p}}}\right)^{n_{r}-1} .
\end{aligned}
$$

In Eq. (2.35) $k_{\mathrm{p}}$ is the pivot scale [already introduced in Eq. (2.2)] at which the spectra are normalized. Typical choices of $k_{\mathrm{p}}$ range from $0.05 \mathrm{Mpc}^{-1}$ down to $0.002 \mathrm{Mpc}^{-1}[1-3]$.

Equations (2.1) and (2.2) allow us to determine the spectra of $\Omega_{\mathrm{B}}(k)$ and $\sigma_{\mathrm{B}}(k)$. In the present notations ${ }^{6}$

$$
\begin{aligned}
\left\langle\Omega_{\mathrm{B}}(\vec{k}) \Omega_{\mathrm{B}}(\vec{p})\right\rangle & =\frac{2 \pi^{2}}{k^{3}} \mathcal{P}_{\Omega}(k) \delta^{(3)}(\vec{k}+\vec{p}), \\
\left\langle\sigma_{\mathrm{B}}(\vec{k}) \sigma_{\mathrm{B}}(\vec{p})\right\rangle & =\frac{2 \pi^{2}}{k^{3}} \mathcal{P}_{\sigma}(k) \delta^{(3)}(\vec{k}+\vec{p}),
\end{aligned}
$$

where

$$
\begin{aligned}
& \mathcal{P}_{\Omega}(k)=\mathcal{F}(\varepsilon) \bar{\Omega}_{\mathrm{B} L}^{2}\left(\frac{k}{k_{L}}\right)^{2 \varepsilon}, \\
& \mathcal{P}_{\sigma}(k)=\mathcal{G}(\varepsilon) \bar{\Omega}_{\mathrm{B} L}^{2}\left(\frac{k}{k_{L}}\right)^{2 \varepsilon} .
\end{aligned}
$$

The power spectra of Eq. (2.37) follow from the definitions of $\Omega_{\mathrm{B}}$ and $\sigma_{\mathrm{B}}$ by using the two-point function of the magnetic field, i.e. Eq. (2.1). In fact, according to Eqs. (2.12) and (2.14), $\Omega_{\mathrm{B}}$ and $\sigma_{\mathrm{B}}$ are quadratic in the magnetic field intensity, and, therefore, lead to two modecoupling integrals that have been estimated in the nearly scale-invariant limit (i.e. $\varepsilon<1$ ) where the functions $\mathcal{F}(\varepsilon)$ and $G(\varepsilon)$ are determined to be (see the Appendix)

$$
\begin{aligned}
\mathcal{F}(\varepsilon) & =\frac{4(6-\varepsilon)(2 \pi)^{2 \varepsilon}}{\varepsilon(3-2 \varepsilon) \Gamma^{2}(\varepsilon / 2)} \\
\mathcal{G}(\varepsilon) & =\frac{4\left(188-4 \varepsilon^{2}-66 \varepsilon\right)(2 \pi)^{2 \varepsilon}}{3 \varepsilon(3-\varepsilon)(2 \varepsilon+1) \Gamma^{2}(\varepsilon / 2)}
\end{aligned}
$$

In the opposite limit (i.e. $\varepsilon \gg 1$ ) the mode-coupling integrals arising in the expressions of $\Omega_{\mathrm{B}}$ and $\sigma_{\mathrm{B}}$ are dominated by (small-scale) diffusive effects (see, for instance, $[35,39]$ ) and the power spectra are sensitive to the specific

\footnotetext{
${ }^{6}$ In the present paper the calculations are consistently conducted within the parametrization introduced in Eqs. (2.1) and (2.2). Different authors use slightly different conventions which can be, however, easily translated in the present parametrization. For instance, the magnetic spectral index defined as $n$ in $[14,35,39,40]$ corresponds, in the present notations, to $\varepsilon-3$. In $[14,35,39,40]$ the factor $2 \pi^{2} / k^{3}$ appearing in Eq. (2.1) is omitted. These definitions are clearly conventional but we regard the notation adopted here as preferable since it agrees with the usual way of assigning the power spectrum of curvature perturbations in CMB physics. The other remark is that the magnetic fields considered here are not helical (see, for instance, [41] and references therein). This is not a limitation since it can be easily appreciated that the helical contribution does not affect the scalar modes which are the main subject of the present investigation.
} 
scale of the diffusion (Alfvén) damping. In Eq. (2.37) the quantities

$$
\bar{\Omega}_{\mathrm{B} L}=\frac{\rho_{\mathrm{B} L}}{\bar{\rho}_{\gamma}}, \quad \rho_{\mathrm{B} L}=\frac{B_{L}^{2}}{8 \pi}, \quad \bar{\rho}_{\gamma}=a^{4}(\tau) \rho_{\gamma}(\tau)
$$

have been introduced. Notice that $B_{L}$ measures the magnetic field intensity smoothed over a comoving length scale $L$. In fact, by using a Gaussian window function $e^{-k^{2} L^{2} / 2}$ for each magnetic field intensity, the (real space) magnetic autocorrelation function at two coincident spatial points is

$$
B_{L}^{2}=\left\langle B_{i}(\vec{x}, \tau) B^{i}(\vec{x}, \tau)\right\rangle=A_{\mathrm{B}}\left(\frac{k_{L}}{k_{\mathrm{p}}}\right)^{\varepsilon} \Gamma\left(\frac{\varepsilon}{2}\right)(2 \pi)^{-\varepsilon}
$$

where $k_{L}=2 \pi / L$. Equation (2.40) implies that the amplitude of the magnetic power spectrum appearing in Eqs. (2.1) and (2.2) can be directly characterized in terms of $B_{L}^{2}$ as

$$
A_{\mathrm{B}}=(2 \pi)^{\varepsilon} \frac{B_{L}^{2}}{\Gamma(\varepsilon / 2)}\left(\frac{k_{\mathrm{p}}}{k_{L}}\right)^{\varepsilon} .
$$

It should be mentioned, incidentally, that nearly scaleinvariant magnetic energy spectra can be achieved both in some class of inflationary models [60] and in the case of pre-big-bang models [61]. It is not our purpose here to endorse any specific model. The spirit of this investigation is more modest since we shall be content with understanding if large-scale magnetic fields can be consistently included in the picture of predecoupling physics and in the framework of the usual techniques commonly employed for the description of scalar CMB anisotropies.

On top of the curvature fluctuations on comoving orthogonal hypersurfaces, another class of gauge-invariant quantities is related to the density contrasts both total and partial (i.e. pertaining to each single species of the plasma). The total density contrast on uniform density hypersurfaces can be defined as a functional of the longitudinal degrees of freedom,

$$
\zeta=-\psi-\frac{\mathcal{H}\left(\delta \rho_{\mathrm{t}}+\delta \rho_{\mathrm{B}}\right)}{\rho_{\mathrm{t}}^{\prime}}
$$

which coincides, for wavelengths larger than the Hubble radius, with $\mathcal{R}$. In fact, using the definitions (2.33) and (2.42) in the Hamiltonian constraint of Eq. (2.15), it can be verified that

$$
\zeta=\mathcal{R}+\frac{\nabla^{2} \psi}{12 \pi G\left(\rho_{\mathrm{t}}+p_{\mathrm{t}}\right)} .
$$

Having introduced $\zeta$, related to the total density contrast, it is also useful to introduce the following four variables:

$$
\begin{array}{ll}
\zeta_{\nu}=-\psi+\frac{\delta_{\nu}}{4}, & \zeta_{\gamma}=-\psi+\frac{\delta_{\gamma}}{4}, \\
\zeta_{\mathrm{c}}=-\psi+\frac{\delta_{\mathrm{c}}}{3}, & \zeta_{\mathrm{b}}=-\psi+\frac{\delta_{\mathrm{b}}}{3},
\end{array}
$$

which are interpreted as the density contrasts for each independent fluid on uniform curvature hypersurfaces.

The evolution for the density contrasts described by Eqs. (2.6), (2.7), (2.22), and (2.23) become, in terms of the variables introduced in (2.44),

$$
\begin{array}{ll}
\zeta_{\gamma}^{\prime}=-\frac{\theta_{\gamma \mathrm{b}}}{3}, & \zeta_{\nu}^{\prime}=-\frac{\theta_{\nu}}{3}, \\
\zeta_{\mathrm{c}}^{\prime}=-\frac{\theta_{\mathrm{c}}}{3}, & \zeta_{\mathrm{b}}^{\prime}=-\frac{\theta_{\gamma \mathrm{b}}}{3} .
\end{array}
$$

Equations (2.44) and (2.45) are a useful tool for the discussion of the nonadiabatic solutions of the system as it will now be explicitly shown.

\section{Magnetized nonadiabatic modes}

The inclusion of fully inhomogeneous magnetic fields not only affects the adiabatic mode but also the other (nonadiabatic) modes whose physical features have been investigated in the conventional case (see, for instance, [57,62-66] and references therein). The adiabatic solution obtained in Eqs. (2.28), (2.29), and (2.31) implies, in terms of the quantities defined in Eq. (2.44), that

$$
\zeta_{\nu}=\zeta_{\gamma}=\zeta_{\mathrm{c}}=\zeta_{\mathrm{b}}=\mathcal{R}_{*}-\frac{R_{\gamma}}{4} \Omega_{\mathrm{B}}
$$

Equation (2.46) is not accidental. It implies, in fact, that the entropy fluctuations of the mixture of fluids are vanishing. The entropy fluctuations are indeed defined as

$$
\mathcal{S}_{\mathrm{ij}}=-3\left(\zeta_{\mathrm{i}}-\zeta_{\mathrm{j}}\right),
$$

where the indices run over the four components of the fluid defined previously. Equation (2.46) implies, therefore, that the entropy fluctuations vanish for the adiabatic mode.

The predecoupling system also admits solutions where entropy fluctuations do not vanish. These modes are called nonadiabatic. If there are entropy fluctuations, the perturbation of the total pressure, appearing in Eqs. (2.17) and (2.24), can be written as

$$
\delta_{\mathrm{s}} p_{\mathrm{t}}=c_{\mathrm{s}}^{2} \delta \rho_{\mathrm{t}}+\delta p_{\mathrm{nad}}
$$

where

$$
\delta p_{\text {nad }}=\frac{1}{6 \mathcal{H} \rho_{\mathrm{t}}^{\prime}} \sum_{\mathrm{ij}} \rho_{\mathrm{i}}^{\prime} \rho_{\mathrm{j}}^{\prime}\left(c_{\mathrm{si}}^{2}-c_{\mathrm{sj}}^{2}\right) S_{\mathrm{ij}}, \quad c_{\mathrm{s}}^{2}=\frac{p_{\mathrm{t}}^{\prime}}{\rho_{\mathrm{t}}^{\prime}} .
$$

In Eq. (2.49) $c_{\mathrm{si}}^{2}$ and $c_{\mathrm{sj}}^{2}$ are the sound speeds of each (generic) pair of fluids of the mixture. According to Eq. (2.48), the fluctuation in the total pressure may arise either because of an inhomogeneity in the energy density 
or thanks to the chemical inhomogeneity of the plasma. Chemically inhomogeneous means, in this context, that the plasma is constituted by various fluids with equations of state that are different and that entail necessarily a spatial variation of the sound speed. As a consequence of Eqs. (2.48) and (2.49), the evolution of $\zeta$ can be directly obtained from the evolution of $\delta_{\mathrm{s}} \rho_{\mathrm{t}}$ stemming from the first-order covariant conservation equation:

$$
\begin{aligned}
& \delta_{\mathrm{s}} \rho_{\mathrm{t}}^{\prime}+3 \mathcal{H}\left(\delta_{\mathrm{s}} \rho_{\mathrm{t}}+\delta_{\mathrm{s}} p_{\mathrm{t}}\right)-3 \psi^{\prime}\left(\rho_{\mathrm{t}}+p_{\mathrm{t}}\right)+\left(p_{\mathrm{t}}+\rho_{\mathrm{t}}\right) \theta_{\mathrm{t}} \\
& \quad=\frac{\vec{E} \cdot \vec{J}}{a^{4}} .
\end{aligned}
$$

Neglecting the Ohmic electric field, we do obtain the wanted evolution of $\zeta$, i.e.

$$
\zeta^{\prime}=-\frac{\mathcal{H}}{p_{\mathrm{t}}+\rho_{\mathrm{t}}} \delta p_{\text {nad }}+\frac{\mathcal{H}}{p_{\mathrm{t}}+\rho_{\mathrm{t}}}\left(c_{\mathrm{s}}^{2}-\frac{1}{3}\right) \delta \rho_{\mathrm{B}}-\frac{\theta_{\mathrm{t}}}{3} .
$$

By using Eqs. (2.44) and (2.51) together with the explicit form of the evolution equations in the longitudinal gauge, the analytic form of the nonadiabatic contributions can be obtained. In the following, as an example, the magnetized CDM-radiation mode and the magnetized neutrino-entropy mode will be presented. For the case of the CDM-radiation mode, the solution, in the limit $\tau<\tau_{1}$ and $k \tau<1$, can be written as

$$
\begin{aligned}
\phi & =\phi_{1}\left(\frac{\tau}{\tau_{1}}\right), \quad \psi=\psi_{1}\left(\frac{\tau}{\tau_{1}}\right), \\
\delta_{\gamma} & =\delta_{\nu}=4 \psi_{1}\left(\frac{\tau}{\tau_{1}}\right)-R_{\gamma} \Omega_{\mathrm{B}}, \\
\delta_{\mathrm{c}} & =-\left[\mathcal{S}_{*}+\frac{3}{4} R_{\gamma} \Omega_{\mathrm{B}}\right]+3 \psi_{1}\left(\frac{\tau}{\tau_{1}}\right), \\
\delta_{\mathrm{b}} & =3 \psi_{1}\left(\frac{\tau}{\tau_{1}}\right)-\frac{3}{4} R_{\gamma} \Omega_{\mathrm{B}}, \quad \theta_{\mathrm{c}}=\frac{k^{2} \tau_{1}}{3} \phi_{1}\left(\frac{\tau}{\tau_{1}}\right)^{2}, \\
\theta_{\gamma \mathrm{b}} & =\frac{k^{2} \tau_{1}}{2}\left(\phi_{1}+\psi_{1}\right)\left(\frac{\tau}{\tau_{1}}\right)^{2}+\frac{k^{2} \tau}{4}\left[R_{\nu} \Omega_{\mathrm{B}}-4 \sigma_{\mathrm{B}}\right], \\
\theta_{\nu} & =\frac{k^{2} \tau_{1}}{2}\left(\phi_{1}+\psi_{1}\right)\left(\frac{\tau}{\tau_{1}}\right)^{2}+\frac{k \tau}{4}\left(4 \frac{R_{\gamma}}{R_{\nu}} \sigma_{\mathrm{B}}-\Omega_{\mathrm{B}}\right), \\
\mathcal{F}_{\nu 3} & =\frac{8}{9} k \tau\left[4 \frac{R_{\gamma}}{R_{\nu}} \sigma_{\mathrm{B}}-\Omega_{\mathrm{B}}\right], \\
\sigma_{\nu} & =-\frac{R_{\gamma}}{R_{\nu}} \sigma_{\mathrm{B}}+\frac{k^{2} \tau_{1}^{2}}{6 R_{\nu}}\left(\psi_{1}-\phi_{1}\right)\left(\frac{\tau}{\tau_{1}}\right)^{3},
\end{aligned}
$$

where

$$
\begin{aligned}
& \psi_{1}=\frac{15+4 R_{\nu}}{8\left(15+2 R_{\nu}\right)}\left[\mathcal{S}_{*}+\frac{3}{4} R_{\gamma} \Omega_{\mathrm{B}}\right], \\
& \phi_{1}=\frac{15-4 R_{\nu}}{8\left(15+2 R_{\nu}\right)}\left[\mathcal{S}_{*}+\frac{3}{4} R_{\gamma} \Omega_{\mathrm{B}}\right] .
\end{aligned}
$$

In the case of this solution, the longitudinal fluctuations of the geometry are vanishing for $\tau<\tau_{1}$ and the relevant entropy fluctuations, i.e. $\mathcal{S}_{\mathrm{c} \gamma}$ and $\mathcal{S}_{\mathrm{c} \nu}$, are constant and proportional to $\mathcal{S}_{*}$. In the limit $\Omega_{\mathrm{B}} \rightarrow 0$ and $\sigma_{\mathrm{B}} \rightarrow 0$, the solution (2.52) reduces to the conventional CDM-radiation mode. While in the standard case the octupole moment of the (perturbed) neutrino phase-space distribution vanishes, in the magnetized case it is proportional to $k \tau$ and is negligible for length scales larger than the Hubble radius prior to equality. In analogy with Eq. (2.35), the spectrum of entropy perturbations will be parametrized as

$$
\begin{aligned}
\left\langle\mathcal{S}_{*}(\vec{k}) \mathcal{S}_{*}(\vec{p})\right\rangle & =\frac{2 \pi^{2}}{k^{3}} \mathcal{P}_{\mathcal{S}}(k) \delta^{(3)}(\vec{k}+\vec{p}), \\
\mathcal{P}_{\mathcal{S}}(k) & =A_{\mathcal{S}}\left(\frac{k}{k_{\mathrm{p}}}\right)^{n_{\mathrm{s}}-1}
\end{aligned}
$$

where $k_{\mathrm{p}}$ is the same pivot scale introduced in Eq. (2.35) while $n_{s}$ is the spectral index of the entropy fluctuation. In the case of the magnetized neutrino-entropy mode, the solution can instead be presented as

$$
\begin{aligned}
\phi & =\phi_{0}, \quad \psi=-\frac{\phi_{0}}{2}, \\
\phi_{0} & =\frac{8 R_{\gamma} R_{\nu}}{3\left(4 R_{\nu}+15\right)}\left[\tilde{\mathcal{S}}_{*}-\frac{3}{R_{\nu}} \sigma_{\mathrm{B}}+\frac{3}{4} \Omega_{\mathrm{B}}\right], \\
\delta_{\gamma} & =-2 \phi_{0}+\frac{4}{3} \tilde{\mathcal{S}}_{*} R_{\nu}-\Omega_{\mathrm{B}} R_{\gamma}, \\
\delta_{\nu} & =-2 \phi_{0}-\frac{4}{3} \tilde{\mathcal{S}}_{*} R_{\gamma}-\Omega_{\mathrm{B}} R_{\gamma}, \\
\delta_{\mathrm{b}} & =\delta_{\mathrm{c}}=-\frac{3}{2} \phi_{0}-\frac{3}{4} \Omega_{\mathrm{B}} R_{\gamma}, \quad \theta_{\mathrm{c}}=\frac{k^{2} \tau}{2} \phi_{0}, \\
\theta_{\gamma \mathrm{b}} & =\frac{k^{2} \tau}{4}\left[2 \phi_{0}-\frac{4}{3} \tilde{\mathcal{S}}_{*} R_{\nu}+R_{\nu} \Omega_{\mathrm{B}}-4 \sigma_{\mathrm{B}}\right], \\
\theta_{\nu} & =\frac{k^{2} \tau}{4}\left[2 \phi_{0}-\frac{4}{3} \tilde{\mathcal{S}}_{*}\left(R_{\gamma}+1\right)+4 \frac{R_{\gamma}}{R_{\nu}} \sigma_{\mathrm{B}}-\Omega_{\mathrm{B}} R_{\gamma}\right] \\
\sigma_{\nu} & =-\frac{R_{\gamma}}{R_{\nu}} \sigma_{\mathrm{B}}-\frac{k^{2} \tau^{2}}{4 R_{\nu}} \phi_{0} .
\end{aligned}
$$

In the case of the neutrino-entropy mode, $\mathcal{S}_{\nu \gamma} \neq 0$ and it is proportional to a constant that has been denoted in Eq. (2.55) as $\tilde{\mathcal{S}}_{*}(k)$; the spectrum of $\tilde{\mathcal{S}}_{*}(k)$ can be defined in full analogy with Eq. (2.54). An interesting feature of the magnetized neutrino-entropy mode (common to the conventional case when magnetic fields are absent) is that the curvature perturbations on comoving orthogonal hypersurfaces [i.e. $\mathcal{R}$ defined in Eq. (2.33)] vanish for wavelengths larger than the Hubble radius before equality. This statement can be verified by using Eq. (2.55) in Eq. (2.33) with the result that $\mathcal{R}=-\psi-\phi / 2=0$ for $k \tau \ll 1$. 


\section{HIGHER-ORDER TIGHT COUPLING EXPANSION}

After having taken the derivative with respect to the conformal time $\tau$ of the first relation appearing in Eq. (2.6), Eq. (2.9) can be used to eliminate the baryonphoton velocity; the result of this manipulation is

$$
\begin{aligned}
\delta_{\gamma}^{\prime \prime}+ & \frac{\mathcal{H} R_{\mathrm{b}}}{R_{\mathrm{b}}+1} \delta_{\gamma}^{\prime}+\frac{k^{2}}{3\left(R_{\mathrm{b}}+1\right)} \delta_{\gamma} \\
= & 4\left[\psi^{\prime \prime}+\frac{\mathcal{H} R_{\mathrm{b}}}{R_{\mathrm{b}}+1} \psi^{\prime}-\frac{k^{2}}{3} \phi\right] \\
& +\frac{k^{2}}{3\left(R_{\mathrm{b}}+1\right)}\left[4 \sigma_{\mathrm{B}}-\Omega_{\mathrm{B}}\right]
\end{aligned}
$$

where, according to Eq. (2.12), the Lorentz force has been traded for a combination of the magnetic energy density and of the normalized anisotropic stress. Equation (3.1) stems directly from the zeroth order in the tight coupling expansion.

To discuss the polarization, we have to go (at least) to first order in the tight coupling expansion. For this purpose, it is appropriate to introduce the evolution equations of the brightness perturbations of the $I, Q$, and $U$ Stokes parameters characterizing the radiation field. Since the Stokes parameters $Q$ and $U$ are not invariant under rotations about the axis of propagation, the degree of polarization $P=$ $\left(Q^{2}+U^{2}\right)^{1 / 2}$ is customarily introduced $[6,67]$. The relevant brightness perturbations will then be denoted as $\Delta_{\mathrm{I}}$, $\Delta_{\mathrm{P}}$. This description reproduces, to zeroth order in the tight coupling expansion, the fluid equations that have been presented in Sec. II to set initial conditions prior to equality. For instance, the photon density contrast and the divergence of the photon peculiar velocity are related, respectively, to the monopole and to the dipole of the brightness perturbation of the intensity field, i.e. $\delta_{\gamma}=$ $4 \Delta_{\mathrm{I} 0}$ and $\theta_{\gamma}=3 k \Delta_{\mathrm{I} 1}$. The evolution equations of the brightness perturbations can then be written, within the conventions set by Eqs. (2.3) and (2.5), as

$$
\begin{gathered}
\Delta_{\mathrm{I}}^{\prime}+\left(i k \mu+\kappa^{\prime}\right) \Delta_{\mathrm{I}}+i k \mu \phi \\
=\psi^{\prime}+\kappa^{\prime}\left[\Delta_{\mathrm{I} 0}+\mu v_{\mathrm{b}}-\frac{1}{2} P_{2}(\mu) S_{\mathrm{P}}\right] \\
\Delta_{\mathrm{P}}^{\prime}+\left(i k \mu+\kappa^{\prime}\right) \Delta_{\mathrm{P}}=\frac{\kappa^{\prime}}{2}\left[1-P_{2}(\mu)\right] S_{\mathrm{P}} \\
v_{\mathrm{b}}^{\prime}+\mathcal{H} v_{\mathrm{b}}+i k \phi+\frac{i k}{4 R_{\mathrm{b}}}\left[\Omega_{\mathrm{B}}-4 \sigma_{\mathrm{B}}\right] \\
+\frac{\kappa^{\prime}}{R_{\mathrm{b}}}\left(v_{\mathrm{b}}+3 i \Delta_{\mathrm{I} 1}\right)=0 .
\end{gathered}
$$

Equation (3.4) is nothing but the second relation obtained in Eq. (2.7), having introduced the quantity $i k v_{\mathrm{b}}=\theta_{\mathrm{b}}$. The source terms appearing in Eqs. (3.2) and (3.3) include a dependence on $P_{2}(\mu)=\left(3 \mu^{2}-1\right) / 2\left[P_{\ell}(\mu)\right.$ denotes, in this framework, the $\ell$ th Legendre polynomial]; $\mu=\hat{k} \cdot \hat{n}$ is simply the projection of the Fourier wave number on the direction of the photon momentum. In Eqs. (3.2) and (3.3) the source term $S_{\mathrm{P}}$ is defined as

$$
S_{\mathrm{P}}(k, \tau)=\Delta_{\mathrm{I} 2}(k, \tau)+\Delta_{\mathrm{P} 0}(k, \tau)+\Delta_{\mathrm{P} 2}(k, \tau) .
$$

The evolution of the longitudinal fluctuations is dictated by Eq. (2.16) and by Eqs. (2.15), (2.16), (2.17), and (2.18). According to the definitions used to derive Eqs. (3.2), (3.3), and (3.4), the $\ell$ th multipole of the brightness perturbations is defined as

$$
\begin{aligned}
& \int_{-1}^{1} P_{\ell}(\mu) \Delta_{\mathrm{I}}(k, \mu, \tau) d \mu=2(-i)^{\ell} \Delta_{\mathrm{I} \ell}(k, \tau), \\
& \int_{-1}^{1} P_{\ell}(\mu) \Delta_{\mathrm{P}}(k, \mu, \tau) d \mu=2(-i)^{\ell} \Delta_{\mathrm{P} \ell}(k, \tau) .
\end{aligned}
$$

Equations (3.2) and (3.3) can now be expanded in powers of $\tau_{\mathrm{c}}=\left|1 / \kappa^{\prime}\right|$. In particular, we can write

$$
\begin{aligned}
\Delta_{\mathrm{I} \ell}(k, \tau) & =\bar{\Delta}_{\mathrm{I} \ell}(k, \tau)+\tau_{\mathrm{c}} \delta_{\mathrm{I} \ell}(k, \tau), \\
\Delta_{\mathrm{P} \ell}(k, \tau) & =\bar{\Delta}_{\mathrm{P} \ell}(k, \tau)+\tau_{\mathrm{c}} \delta_{\mathrm{P} \ell}(k, \tau), \\
v_{\mathrm{b}} & =\bar{v}_{\mathrm{b}}(k, \tau)+\tau_{\mathrm{c}} \delta_{v_{\mathrm{b}}}(k, \tau) .
\end{aligned}
$$

Equations (3.2) and (3.3) will be preliminarily phrased in terms of a Boltzmann hierarchy coupling together the different multipoles of the brightness perturbations. Then the obtained equations will be expanded according to (3.7). The result of the first step of this procedure is

$$
\begin{gathered}
\Delta_{\mathrm{I} 0}^{\prime}+k \Delta_{\mathrm{I} 1}=\psi^{\prime} \\
\Delta_{\mathrm{I} 1}^{\prime}+\frac{2}{3} k \Delta_{\mathrm{I} 2}-\frac{k}{3} \Delta_{\mathrm{I} 0}=\frac{k}{3} \phi-\kappa^{\prime}\left[\Delta_{\mathrm{I} 1}+\frac{v_{\mathrm{b}}}{3 i}\right] \\
\Delta_{\mathrm{I} 2}^{\prime}+\frac{3}{5} k \Delta_{\mathrm{I} 3}-\frac{2}{5} k \Delta_{\mathrm{I} 1}=-\frac{\kappa^{\prime}}{10}\left[9 \Delta_{\mathrm{I} 2}-\left(\Delta_{\mathrm{P} 0}+\Delta_{\mathrm{P} 2}\right)\right]
\end{gathered}
$$

$\Delta_{\mathrm{I} \ell}^{\prime}+\kappa^{\prime} \Delta_{\mathrm{I} \ell}=\frac{k}{2 \ell+1}\left[\ell \Delta_{\mathrm{I}(\ell-1)}-(\ell+1) \Delta_{\mathrm{I}(\ell+1)}\right], \quad \ell>2$.

Equations (3.8), (3.9), and (3.10) are obtained, respectively, by multiplying each side of Eq. (3.2) by $P_{0}(\mu), P_{1}(\mu)$, and $P_{2}(\mu)$ and by integrating over $\mu$ with the help of Eq. (3.6). The same calculation can be repeated in the case of Eq. (3.3); the result is

$$
\begin{gathered}
\Delta_{\mathrm{P} 0}^{\prime}+k \Delta_{\mathrm{P} 1}=\frac{\kappa^{\prime}}{2}\left[\Delta_{\mathrm{P} 2}+\Delta_{\mathrm{I} 2}-\Delta_{\mathrm{P} 0}\right] \\
\Delta_{\mathrm{P} 1}^{\prime}+\frac{2}{3} k \Delta_{\mathrm{P} 2}-\frac{k}{3} \Delta_{\mathrm{P} 0}=-\kappa^{\prime} \Delta_{\mathrm{P} 1}
\end{gathered}
$$




$$
\Delta_{\mathrm{P} 2}^{\prime}+\frac{3}{5} k \Delta_{\mathrm{P} 3}-\frac{2}{5} k \Delta_{\mathrm{P} 1}=-\frac{\kappa^{\prime}}{10}\left[9 \Delta_{\mathrm{P} 2}-\left(\Delta_{\mathrm{P} 0}+\Delta_{\mathrm{I} 2}\right)\right],
$$

$\Delta_{\mathrm{P} \ell}^{\prime}+\kappa^{\prime} \Delta_{\mathrm{P} \ell}=\frac{k}{2 \ell+1}\left[\ell \Delta_{\mathrm{P}(\ell-1)}-(\ell+1) \Delta_{\mathrm{P}(\ell+1)}\right], \quad \ell>2$.

Having derived Eqs. (3.8), (3.9), (3.10), (3.11), (3.12), (3.13), (3.14), and (3.15), the system can now be expanded to the wanted order in $\tau_{\mathrm{c}}$. As anticipated, to zeroth order in the tight coupling expansion, Eq. (3.1) can be reobtained. In fact, to lowest order in $\tau_{\mathrm{c}}$,

$$
\overline{\boldsymbol{v}}_{\mathrm{b}}(k, \tau)=-3 i \bar{\Delta}_{\mathrm{I} 1}(k, \tau), \quad \bar{\Delta}_{\mathrm{I} 0}^{\prime}+k \bar{\Delta}_{\mathrm{I} 1}=\psi^{\prime},
$$

while, from Eqs. (3.12), (3.13), (3.14), and (3.15),

$$
\bar{\Delta}_{\mathrm{P} 0}(k, \tau)=0, \quad \ell \geq 0, \quad \bar{\Delta}_{\mathrm{I} \ell}(k, \tau)=0, \quad \ell \geq 2 .
$$

Summing up Eq. (3.8) (multiplied by 3i) with Eq. (3.4) (multiplied by $R_{\mathrm{b}}$ ) and eliminating, from the obtained relation, $\bar{\Delta}_{\mathrm{I} 1}$ in favor of $\bar{\Delta}_{\mathrm{I} 0}^{\prime}$ [see Eq. (3.16)] the decoupled expression for the evolution of the zeroth-order monopole becomes, as expected,

$$
\begin{aligned}
& \bar{\Delta}_{\mathrm{I} 0}^{\prime \prime}+\frac{\mathcal{H} R_{\mathrm{b}}}{R_{\mathrm{b}}+1} \bar{\Delta}_{\mathrm{I} 0}^{\prime}+\frac{k^{2}}{3\left(R_{\mathrm{b}}+1\right)} \bar{\Delta}_{\mathrm{I} 0} \\
& =\left(\psi^{\prime \prime}+\frac{\mathcal{H} R_{\mathrm{b}}}{1+R_{\mathrm{b}}} \psi^{\prime}-\frac{k^{2}}{3} \phi\right)+\frac{k^{2}\left(4 \sigma_{\mathrm{B}}-\Omega_{\mathrm{B}}\right)}{3\left(R_{\mathrm{b}}+1\right)},
\end{aligned}
$$

which is the same as Eq. (3.1) if we recall that $4 \bar{\Delta}_{\mathrm{I} 0}=\delta_{\gamma}$. The presence of the magnetic field modifies the evolution of the zeroth-order monopole and, consequently, according to Eq. (3.16), the zeroth-order dipole. Such a modification also implies an effect on the polarization. In fact, to first order in the tight coupling expansion, $\Delta_{\mathrm{P} 0} \neq 0$ and it is proportional to the zeroth-order dipole. Recalling the notations of Eq. (3.7) and expanding Eqs. (3.8), (3.9), (3.10), (3.11), (3.12), (3.13), (3.14), and (3.15) to first order in $\tau_{\mathrm{c}}$, it can be verified that

$$
\begin{gathered}
\delta_{\mathrm{P} 0}(k, \tau)=\frac{5}{4} \delta_{\mathrm{I} 2}(k, \tau), \quad \delta_{\mathrm{P} 2}(k, \tau)=\frac{1}{4} \delta_{\mathrm{I} 2}(k, \tau), \\
\delta_{\mathrm{I} 2}(k, \tau)=\frac{8}{15} k \bar{\Delta}_{\mathrm{I} 1}(k, \tau),
\end{gathered}
$$

which also implies

$$
\Delta_{\mathrm{I} 2}(k, \tau) \simeq \tau_{\mathrm{c}} \delta_{\mathrm{I} 2}(k, \tau) \simeq \frac{8}{15} k \tau_{\mathrm{c}} \bar{\Delta}_{\mathrm{I} 1}(k, \tau) .
$$

Since $\tau_{\mathrm{c}}$ grows very fast during decoupling, to have a better quantitative estimate of the effect, the procedure outlined in [6] can be followed. By taking the first derivative of the definition of $S_{\mathrm{P}}$ and by using Eqs. (3.11) and (3.15) we can get, directly, the following evolution equation for $S_{\mathrm{P}}$,

$$
S_{\mathrm{P}}^{\prime}+\frac{3}{10} \kappa^{\prime} S_{\mathrm{P}}=\frac{2}{5} k \bar{\Delta}_{\mathrm{I} 1} \text {. }
$$

Equation (3.21) can be used to estimate the source term in Eq. (3.3) and, therefore, the polarization can be determined as

$$
\Delta_{\mathrm{P}}\left(k, \tau_{\mathrm{dec}}\right)=-\frac{3}{10}\left(1-\mu^{2}\right) \bar{\Delta}_{\mathrm{I} 1}\left(\tau_{\mathrm{dec}}\right) k \sigma_{\mathrm{dec}} I_{1},
$$

where $\sigma_{\mathrm{dec}} \simeq 70-80 \mathrm{Mpc}$ is the width of the visibility function $\kappa^{\prime} e^{-\kappa}$ which has a Gaussian form [68]. The quantity $I_{1}$ appearing in Eq. (3.22) is

$$
I_{1}=\int_{0}^{\infty} e^{-(7 / 10) \kappa} d \kappa \int_{1}^{\infty} \frac{d x}{x} e^{-(3 / 10) \kappa x} \simeq 1.719 .
$$

Let us now perform an oversimplified estimate. Let us ignore neutrinos and magnetic fields and let us compute out of Eq. (3.2) the ordinary Sachs-Wolfe contribution assuming a constant adiabatic mode that propagates smoothly through the matter-radiation transition. The constant mode will be $\psi_{\mathrm{m}} \simeq-(3 / 5) \mathcal{R}_{*}$ where $\mathcal{R}_{*}$ is the constant mode of curvature fluctuations. Then, from Eqs. (3.18), (3.22), and (3.23),

$\Delta_{\mathrm{I}}\left(k, \tau_{\mathrm{dec}}\right) \simeq \frac{\psi_{\mathrm{m}}}{3} \cos \left(k c_{\mathrm{sb}} \tau_{\mathrm{dec}}\right)$

$\Delta_{\mathrm{P}}\left(k, \tau_{\mathrm{dec}}\right)=-0.17\left(1-\mu^{2}\right)\left(\sigma_{\mathrm{dec}} k c_{\mathrm{sb}}\right) \psi_{\mathrm{m}} \sin \left(k c_{\mathrm{sb}} \tau_{\mathrm{dec}}\right)$,

which implies that the cross-correlation between temperature and polarization oscillates as $\sin \left(2 k c_{\mathrm{sb}} \tau_{\mathrm{dec}}\right)$.

It is finally interesting to compute the expression of the characteristic damping scale of the fluctuations arising from the dispersion relations. From Eq. (3.4), by taking the Laplace transform, i.e. $v_{\mathrm{b}}(k, \tau)=e^{i \int \omega\left(\tau^{\prime}\right) d \tau^{\prime}} v_{\mathrm{b}}(k, \omega)$ (and similarly for the brightness perturbations), we will have that the following relation holds to second order in the tight coupling expansion:

$$
\begin{aligned}
v_{\mathrm{b}}(k, \omega)= & -3 i \Delta_{\mathrm{I} 1}(k, \omega)\left[1-i \frac{\omega R_{\mathrm{b}}}{\left|\kappa^{\prime}\right|}-\frac{\omega^{2} R_{\mathrm{b}}^{2}}{\left|\kappa^{\prime}\right|^{2}}\right]+\mathcal{O}(\phi) \\
& +\mathcal{O}\left(\Omega_{\mathrm{B}}, \sigma_{\mathrm{B}}\right) .
\end{aligned}
$$

In the standard derivation of the dispersion relations, the metric fluctuations are neglected. This procedure is also justified in the present case provided the spectrum of the magnetic energy density is not too steep in frequency. In practice this is the case for $\varepsilon$ sufficiently smaller than 1 . In this case the magnetic contribution can also be neglected. In this situation, we can also Laplace transform the other equations (i.e. for the monopole, dipole, and quadrupole). Then the first-order tight coupling expressions can be used in Eqs. (3.9) and (3.25) to eliminate $v_{\mathrm{b}}$. The resulting expression will then be 


$$
-\omega^{2}\left(R_{\mathrm{b}}+1\right)+\frac{k^{2}}{3}+i \frac{\omega}{\left|\kappa^{\prime}\right|}\left[\frac{16}{45} k^{2}+\omega^{2} R_{\mathrm{b}}^{2}\right]=0,
$$

which implies that $\omega=\omega_{1}+i \omega_{2}$ where

$$
\begin{gathered}
\omega_{1}=\frac{k}{3\left(1+R_{\mathrm{b}}\right)}, \quad \omega_{2}=\frac{k^{2}}{k_{\mathrm{d}}}, \\
k_{\mathrm{d}}^{-2}=\int_{0}^{\tau} \frac{d \tilde{\tau}}{2 \kappa^{\prime}(\tilde{\tau})}\left[\frac{16}{45\left(R_{\mathrm{b}}(\tilde{\tau})+1\right.}+\frac{R_{\mathrm{b}}^{2}(\tilde{\tau})}{3\left(1+R_{\mathrm{b}}(\tilde{\tau})\right)^{2}}\right] .
\end{gathered}
$$

In practice, for the typical set of parameters introduced around Eq. (2.10), $k_{\mathrm{d}}^{-1} \simeq 3 \mathrm{Mpc}$, which sets, grossly speaking, the typical scale of diffusion damping.

\section{NUMERICAL ANALYSIS}

The evolution equations in the tight coupling approximation will now be integrated numerically. The normalization of the numerical calculation is enforced by evaluating, analytically, the Sachs-Wolfe plateau and by deducing, for a given set of spectral indices of curvature and entropy perturbations, the amplitude of the power spectra at the pivot scale. Here is an example of this strategy. The SW plateau can be estimated analytically from the evolution equation of $\mathcal{R}$ (or $\zeta$ ) by using the technique of the transfer matrix appropriately generalized to the case where, on top of the adiabatic and nonadiabatic contributions, the magnetic fields are consistently taken into account. The result of this calculation can be expressed, for $\ell<30$, as [7]

$$
\begin{aligned}
C_{\ell}= & {\left[\frac{\mathcal{A}_{\mathcal{R}}}{25} Z_{1}\left(n_{r}, \ell\right)+\frac{9}{100} R_{\gamma}^{2} \bar{\Omega}_{\mathrm{B} L}^{2} Z_{2}(\epsilon, \ell)\right.} \\
& -\frac{4}{25} \sqrt{\mathcal{A}_{\mathcal{R}} \mathcal{A}_{\mathcal{S}}} Z_{1}\left(n_{\mathrm{rs}}, \ell\right) \cos \gamma_{\mathrm{rs}} \\
& +\frac{4}{25} \mathcal{A}_{\mathcal{S}} Z_{1}\left(n_{\mathrm{s}}, \ell\right)-\frac{3}{25} \sqrt{\mathcal{A}_{\mathcal{R}}} R_{\gamma} \bar{\Omega}_{\mathrm{B} L} Z_{3}\left(n_{\mathrm{r}}, \varepsilon, \ell\right) \\
& \left.\times \cos \gamma_{\mathrm{br}}+\frac{6}{25} \sqrt{\mathcal{A}_{\mathcal{S}}} R_{\gamma} \bar{\Omega}_{\mathrm{B} L} Z_{3}\left(n_{\mathrm{s}}, \varepsilon, \ell\right) \cos \gamma_{\mathrm{bs}}\right],
\end{aligned}
$$

where the functions $Z_{1}, Z_{2}$, and $Z_{3}$ are

$$
\begin{aligned}
& Z_{1}(n, \ell)=\frac{\pi^{2}}{4}\left(\frac{k_{0}}{k_{\mathrm{p}}}\right)^{n-1} 2^{n} \frac{\Gamma(3-n) \Gamma\left(\ell+\frac{n-1}{2}\right)}{\Gamma^{2}\left(2-\frac{n}{2}\right) \Gamma\left(\ell+\frac{5}{2}-\frac{n}{2}\right)}, \\
& Z_{2}(\varepsilon, \ell)=\frac{\pi^{2}}{2} 2^{2 \varepsilon} \mathcal{F}(\varepsilon)\left(\frac{k_{0}}{k_{L}}\right)^{2 \varepsilon} \frac{\Gamma(2-2 \varepsilon) \Gamma(\ell+\varepsilon)}{\Gamma^{2}\left(\frac{3}{2}-\varepsilon\right) \Gamma(\ell+2-\varepsilon)},
\end{aligned}
$$

$$
\begin{aligned}
Z_{3}(n, \varepsilon, \ell)= & \frac{\pi^{2}}{4} 2^{\varepsilon} 2^{(n+1 / 2)} \sqrt{\mathcal{F}(\varepsilon)}\left(\frac{k_{0}}{k_{L}}\right)^{\varepsilon}\left(\frac{k_{0}}{k_{\mathrm{p}}}\right)^{(n+1 / 2)} \\
& \times \frac{\Gamma\left(\frac{5}{2}-\varepsilon-\frac{n}{2}\right) \Gamma\left(\ell+\frac{\varepsilon}{2}+\frac{n}{4}-\frac{1}{4}\right)}{\Gamma^{2}\left(\frac{7}{4}-\frac{\varepsilon}{2}-\frac{n}{4}\right) \Gamma\left(\frac{9}{4}+\ell-\frac{\varepsilon}{2}-\frac{n}{4}\right)} .
\end{aligned}
$$

We recall the meaning of the various spectral indices appearing in Eqs. (4.2), (4.3), and (4.4). The spectral index $\varepsilon$ has been introduced in Eq. (2.2) [see also Eq. (2.37) for the magnetic energy spectrum]; $k_{0}=\tau_{0}^{-1}$ where $\tau_{0}$ is the present observation time. In Eqs. (4.2), (4.3), and (4.4), $n$ stands either for $n_{\mathrm{r}}$ [i.e. the spectral index of the adiabatic mode defined in Eq. (2.35)], $n_{\mathrm{s}}$ [i.e. the spectral index of the entropic mode defined in Eq. (2.54)], or for $n_{\mathrm{rs}}$ (i.e. the spectral index of the cross-correlation). In principle, one could also introduce a different spectral index for each cross-correlation. However, following for instance [66] we will assume that the spectrum of the cross-correlation is solely determined in terms of the spectrum of the autocorrelations, i.e., for instance, $n_{\mathrm{rs}}=\left(n_{\mathrm{r}}+n_{\mathrm{s}}\right) / 2$. In Eq. (4.1), $\gamma_{\mathrm{rs}}, \gamma_{\mathrm{br}}$, and $\gamma_{\mathrm{sb}}$ are the correlation angles. From the request that the SW plateau is dominated by the adiabatic mode, it is possible to constrain $\bar{\Omega}_{\mathrm{B} L}$ and, consequently, $B_{L}$ [see, indeed, Eq. (2.39)]. The nature of the bound, however, depends on the parameters that enter Eq. (4.1). In the absence of magnetic and nonadiabatic contributions, Eqs. (4.1) and (4.2) imply that for $n_{r}=1$ (Harrison-Zeldovich spectrum) $\quad \ell(\ell+1) C_{\ell} / 2 \pi=$ $\mathcal{A}_{\mathcal{R}} / 25$ and WMAP data [12] would demand that $\mathcal{A}_{\mathcal{R}}=$ $2.65 \times 10^{-9}$.

If the SW plateau is determined by an adiabatic component supplemented by a (subleading) nonadiabatic contribution, both correlated with the magnetic field intensity, the obtainable bound may not be so constraining (even well above the $n G$ range) due to the proliferation of parameters. A possible strategy is therefore to fix the parameters of the adiabatic mode to the values determined by WMAP-3 and then explore the effect of a magnetized contribution which is not correlated with the adiabatic mode. This implies, in Eq. (4.1), that $\mathcal{A}_{\mathcal{S}}=0$ and $\gamma_{\mathrm{br}}=\pi / 2$. Under this assumption, in Fig. 1 the bounds on $B_{L}$ are illustrated. The nature of the constraint depends, in this case, both on the amplitude of the protogalactic field (at the present epoch and smoothed over a typical comoving scale $L=2 \pi / k_{L}$ ) and upon its spectral slope, i.e. $\varepsilon$. In the case $\varepsilon<0.5$, the magnetic energy spectrum is nearly scale invariant. In this case, diffusivity effects are negligible (see, for instance, $[14,15])$. As already discussed, if $\varepsilon \gg 1$ the diffusivity effects (both thermal and magnetic) dominate the modecoupling integral that leads to the magnetic energy spectrum $[14,15]$. In Fig. 1 (left plot) the magnetic field intensity should be below the different curves if the adiabatic contribution dominates the SW plateau. Different choices of the pivot scale $k_{\mathrm{p}}$ and of the smoothing scale $k_{L}$ are also illustrated. In Fig. 1 (left plot) the scalar spectral index is fixed to $n_{r}=0.951$ [3]. In the right plot the two curves 

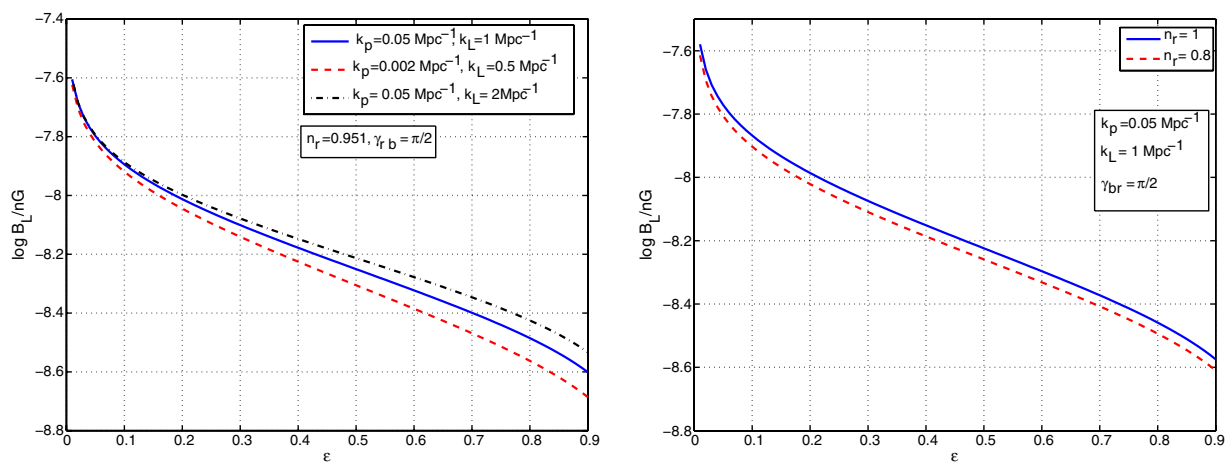

FIG. 1 (color online). Bounds on the protogalactic field intensity as a function of the magnetic spectral index $\varepsilon$ for different values of the parameters defining the adiabatic contribution to the SW plateau.

corresponding, respectively, to $n_{\mathrm{r}}=0.8$ and $n_{\mathrm{r}}=1$ are reported. If $\varepsilon<0.2$ the bounds are comparatively less restrictive than in the case $\varepsilon \simeq 0$.9. The cause of this occurrence is that we are here just looking at the largest wavelengths of the problem. As it will become clear in a moment, intermediate scales will be more sensitive to the presence of fully inhomogeneous magnetic fields.

According to Fig. 1, for a given value of the magnetic spectral index and of the scalar spectral index, the amplitude of the magnetic field has to be sufficiently small not to affect the dominant adiabatic nature of the SW plateau. Therefore, Fig. 1 (as well as other similar plots) can be used to normalize the numerical calculations for the power spectra of the brightness perturbations, i.e.

$$
\begin{gathered}
\frac{k^{3}}{2 \pi^{2}}\left|\Delta_{\mathrm{I}}(k, \tau)\right|^{2}, \quad \frac{k^{3}}{2 \pi^{2}}\left|\Delta_{\mathrm{P}}(k, \tau)\right|^{2}, \\
\frac{k^{3}}{2 \pi^{2}}\left|\Delta_{\mathrm{I}}(k, \tau) \Delta_{\mathrm{P}}(k, \tau)\right| .
\end{gathered}
$$

Let us then assume, for consistency with the cases reported in Fig. 1, that we are dealing with the situation where the magnetic field is not correlated with the adiabatic mode. It is then possible to choose a definite value of the magnetic spectral index (for instance, $\epsilon=0.1$ ) and a definite value of the adiabatic spectral index, i.e. $n_{\mathrm{r}}$ (for instance, $n_{\mathrm{r}}=$ 0.951 , in agreement with [3]). By using the SW plateau, the normalization can be chosen in such a way that the adiabatic mode dominates over the magnetic contribution. In the mentioned case, Fig. 1 implies $B_{L}<1.14 \times 10^{-8} \mathrm{G}$ for a pivot scale $k_{p}=0.002 \mathrm{Mpc}^{-1}$. Since the relative weight of the power spectra given in Eqs. (2.35) and (2.37) is fixed, it is now possible to set initial conditions for the adiabatic mode according to Eqs. (2.28), (2.30), (2.31), and (2.34) deep in the radiation-dominated phase. The initial time of integration will be chosen as $\tau_{\mathrm{i}}=10^{-6} \tau_{1}$ in the notations discussed in Eq. (2.25). According to Eq. (2.26), this choice implies that $\tau_{\mathrm{i}} \ll \tau_{\text {eq }}$.

The power spectra of the brightness perturbations, i.e. Eq. (4.5), can then be computed by numerical integration.
Clearly, the calculation will depend upon the values of $\omega_{\mathrm{m}}$, $\omega_{\mathrm{b}}, \omega_{\mathrm{c}}$, and $R_{\nu}$. We will simply fix these parameters to their fiducial values reported in Eqs. (2.10) [see also (2.11)] and we will take $N_{\nu}=3$ in Eq. (2.32) determining, in this way, the fractional contribution of the neutrinos to the radiation plasma. The first interesting exercise, for the present purposes, is reported in Fig. 2 where, in the left column, the power spectra of the brightness perturbations are illustrated for a wave number $k=0.1 \mathrm{Mpc}^{-1}$; in the right column the power spectra of the same quantities are illustrated for $k=0.2 \mathrm{Mpc}^{-1}$. Concerning the results reported in Fig. 2, different comments are in order:

(i) for $\varepsilon=0.1$ and $n_{\mathrm{r}}=0.951$, the SW plateau imposes $B_{L}<1.14 \times 10^{-8} \mathrm{G}$; from Fig. 2 it follows that a magnetic field of only $30 \mathrm{nG}$ (i.e. marginally incompatible with the SW bound) has a large effect on the brightness perturbations as it can be argued by comparing, in Fig. 2, the dashed curves (corresponding to $30 \mathrm{nG}$ ) to the solid curves which illustrate the case of vanishing magnetic fields;

(ii) the situation where $B_{L}>\mathrm{nG}$ cannot be simply summarized by saying that the amplitudes of the power spectra get larger since there is a combined effect which both increases the amplitudes and shifts slightly the phases of the oscillations;

(iii) from the qualitative point of view, it is still true that the intensity oscillates as a cosine, the polarization as a sine;

(iv) the phases of the cross-correlations are, comparatively, the most affected by the presence of the magnetic field.

The features arising in Figs. 1 and 2 can be easily illustrated for other values of $\epsilon$ and for different choices of the pivot or smoothing scales. The general lesson that can be drawn is that the constraints derived only by looking at the SW plateau are a necessary condition only on the strength of the magnetic field. They are, however, not sufficient to exclude observable effects at smaller scales. This aspect is illustrated in the left plot of Fig. 3 which captures a detail of the cross-correlation. The case when $B_{L}=0$ can still be 

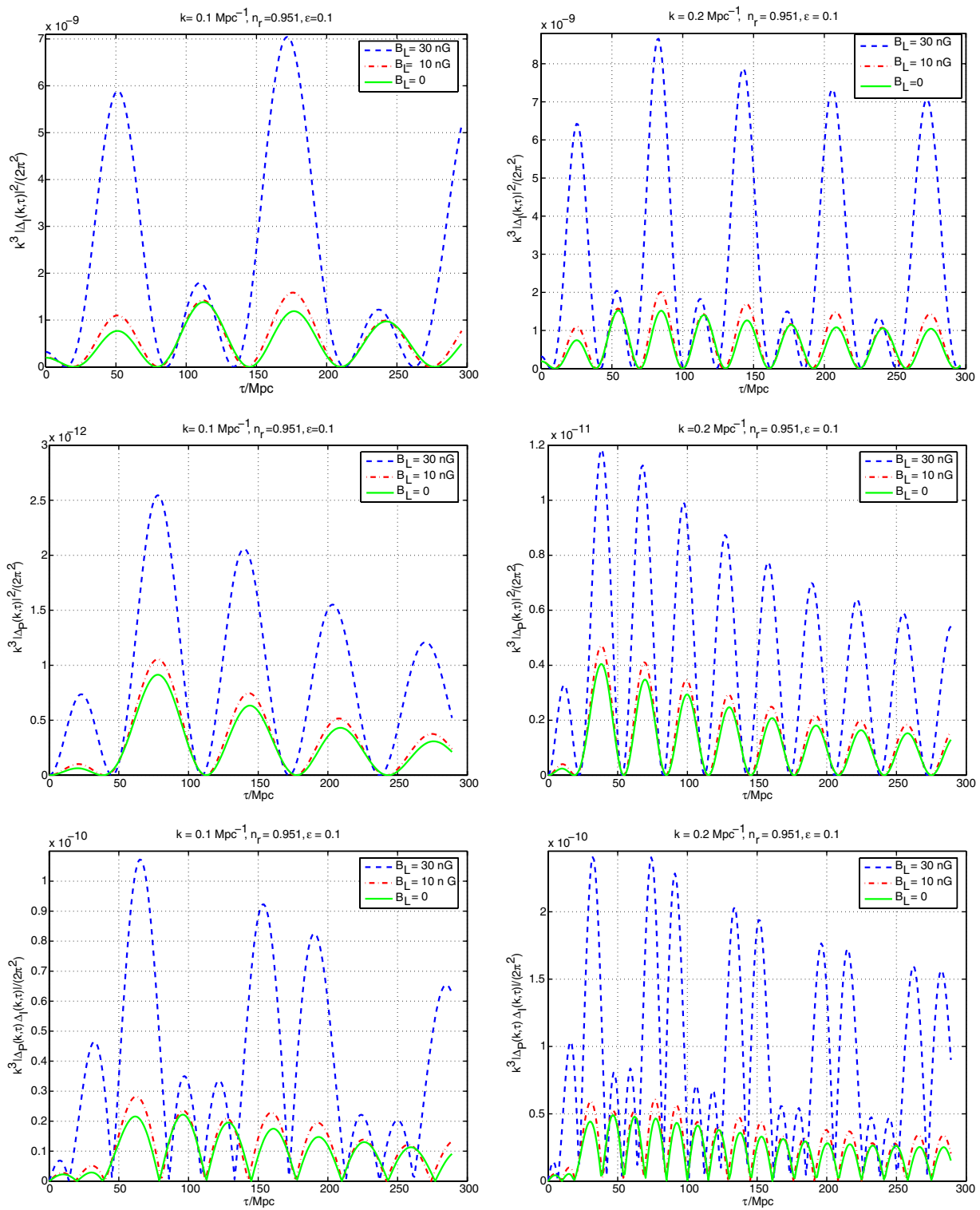

FIG. 2 (color online). The power spectra of the brightness perturbations for two typical wave numbers. The values of the parameters are specified in the legends. The pivot scale is $k_{\mathrm{p}}=0.002 \mathrm{Mpc}^{-1}$ and the smoothing scale is $k_{L}=\mathrm{Mpc}^{-1}$ (see Fig. 1).
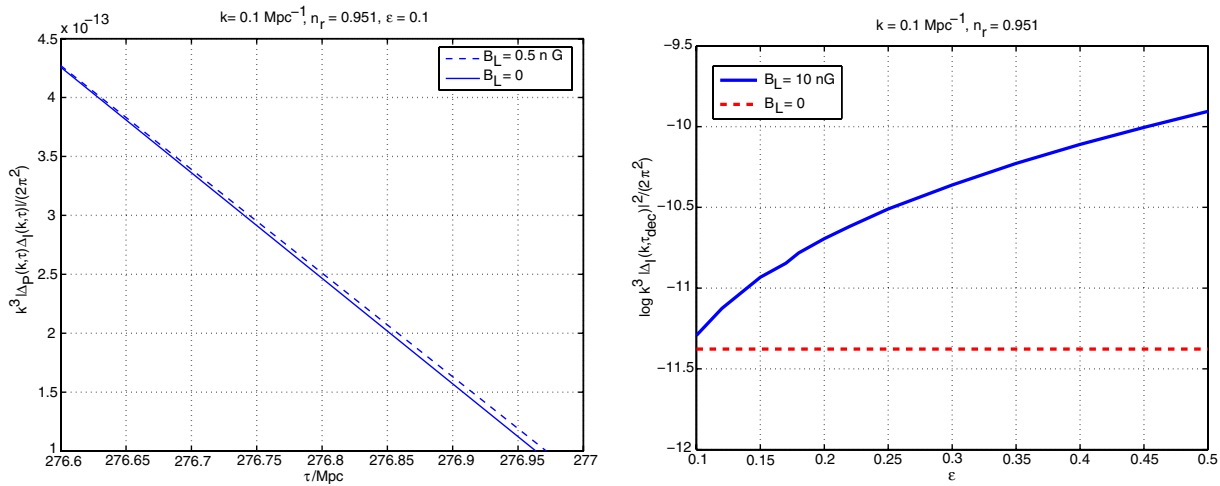

FIG. 3 (color online). A detail of the cross-correlation (left plot). The autocorrelation of the intensity at $\tau_{\text {dec }}$ as a function of $\varepsilon$, i.e. the magnetic spectral index (right plot). 
distinguished from the case $B_{L}=0.5 \mathrm{nG}$. Therefore, recalling that for the same choice of parameters the SW plateau implied that $B_{L}<11.4 \mathrm{nG}$, it is apparent that the intermediate scales lead to more stringent conditions even for nearly scale-invariant spectra of magnetic energy density. For the range of parameters of Fig. 2, we will have that $B_{L}<0.5 \mathrm{nG}$ which is more stringent than the condition deduced from the SW plateau by, roughly, 1 order of magnitude.

Concerning the previous statements, a side remark is in order. The obtained limits follow from the variation of the magnetic field parameters determining the initial conditions of the numerical integration, as thoroughly discussed in Sec. II. Since the other late-time parameters have been fixed to the best fit of WMAP-3, the comments appearing in the previous paragraph only represent a semiquantitative indication which should be corroborated by more appropriate statistical analyses. Nonetheless, the obtained results can be useful in order to build strategies of parameter extraction including, on top of the usual parameters of $\Lambda \mathrm{CDM}$ paradigms, also predecoupling magnetic fields. Such an investigation is beyond the scope of the present paper.

If $\varepsilon$ increases to higher values (but always with $\varepsilon<0.5$ ) by keeping $B_{L}$ (i.e. the strength of the magnetic field smoothed over a typical length scale $L=2 \pi / k_{L}$ ) fixed, the amplitude of the brightness perturbations gets larger in comparison with the case when the magnetic field is ab- sent. This aspect is illustrated in the right plot of Fig. 2 where the logarithm (to base 10) of the intensity autocorrelation is evaluated at a fixed wave number (and at $\tau_{\mathrm{dec}}$ ) as a function of $\varepsilon$. The solid line (corresponding to a $B_{L}=$ $10 \mathrm{nG}$ ) is progressively divergent from the dashed line (corresponding to $B_{L}=0$ ) as $\varepsilon$ increases.

In Fig. 4 the power spectra of the brightness perturbations are reported at $\tau_{\mathrm{dec}}$ and as a function of $k$. In the two plots at the top, the autocorrelation of the intensity is reported for different values of $B_{L}$ (left plot) and for different values of $\varepsilon$ at fixed $B_{L}$ (right plot). In the two plots at the bottom, the polarization power spectra are reported always at $\tau_{\mathrm{dec}}$ and for different values of $B_{L}$ at fixed $\varepsilon$. The position of the first peak of the autocorrelation of the intensity is, approximately, $k_{\mathrm{d}} \simeq 0.017 \mathrm{Mpc}^{-1}$. The position of the first peak of the cross-correlation is, approximately, $3 / 4$ of $k_{\mathrm{d}}$. From this consideration, again, we can obtain that $B_{L}<0.3 \mathrm{nG}$ which is more constraining than the SW condition.

Up to now the adiabatic mode has been considered in detail. We could easily add, however, nonadiabatic modes that are partially correlated with the adiabatic mode. It is rather plausible, in this situation, that, by adding new parameters, the allowed value of the magnetic field may also increase. Similar results can be achieved by deviating from the assumption that the magnetic field and the curvature perturbations are uncorrelated. This aspect can be understood already from the analytical form of the SW
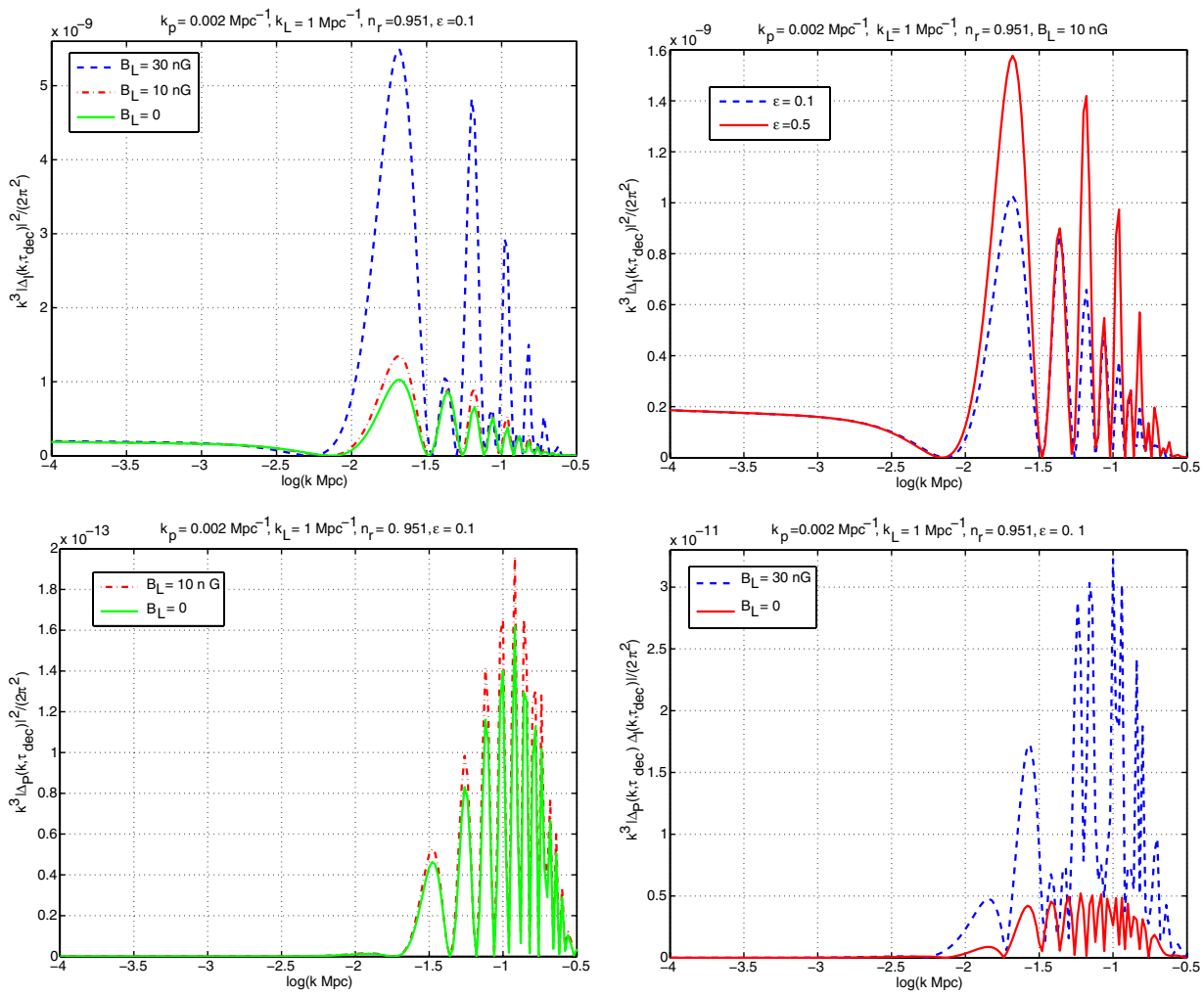

FIG. 4 (color online). The power spectra of the brightness perturbations at $\tau_{\mathrm{dec}}$ for the parameters reported in the legends. 
plateau (4.1). If there is no correlation between the magnetized contribution and the adiabatic contribution, i.e. $\gamma_{\mathrm{br}}=\pi / 2$, the SW plateau will be enhanced in comparison with the case when magnetic fields are absent. The same situation arises when the two components are anticorrelated (i.e. $\cos \gamma_{\mathrm{br}}<0$ ). However, if the fluctuations are positively correlated (i.e. $\cos \gamma_{\mathrm{br}}>0$ ) the crosscorrelation adds negatively to the sum of the two autocorrelations of $\mathcal{R}$ and $\Omega_{\mathrm{B}}$ so that the total result may be an overall reduction of the power with respect to the case $\gamma_{\mathrm{br}}=\pi / 2$.

\section{CONCLUDING REMARKS}

In the recent past, various attempts have been made to constrain large-scale magnetic fields from CMB physics. While interesting results have been obtained for the vector and tensor modes of the geometry, the scalar modes have been, comparatively, less studied. In this study a systematic strategy to tackle this problem has been proposed and developed. The basic inspiration of the present approach can be summarized by stating that the effects of fully inhomogeneous magnetic fields on CMB anisotropies are not univocal. In the standard lore, the only relevant parameters to define the effects of large-scale magnetic fields on $\mathrm{CMB}$ anisotropies are the ones related to the magnetic field itself. For instance, if the magnetic field is uniform the only parameter will be its intensity; if the magnetic field is fully inhomogeneous the only two parameters will be the spectral amplitude and the spectral slope. The point of view of the present investigation is that this way of thinking is ambiguous and, to some extent, not so productive. In fact, the spectral parameters of the magnetic field are certainly a necessary ingredient of the analysis. They are, however, not sufficient to conduct the calculation of the brightness perturbations. The information on the spectral parameters must be complemented by the specific predecoupling initial conditions. According to the results of this investigation, a sound procedure to be followed can be summarized as follows:

(i) include the magnetic fields in the system of scalar modes before matter-radiation equality;

(ii) solve the system in the long wavelength approximation prior to decoupling;

(iii) use the obtained solutions for the numerical evaluation of the brightness perturbations, for instance, in the tight coupling approximation.

The ambiguity mentioned above arises during the first step of the procedure we just outlined. In fact, the inclusion of magnetic fields prior to equality does not lead to a single solution but to different solutions. We will then have quasiadiabatic and quasientropic modes where a magnetized contribution can be accommodated.

In some sense, the present proposal is more conservative than previous attempts: the signature of magnetic fields on CMB physics is not directly accessible but it is mediated by the (supposedly rich) parameter space of the predecoupling initial conditions of CMB anisotropies. This approach is indeed common when mixtures of adiabatic and nonadiabatic modes are studied in the absence of magnetic fields. In the latter case the philosophy is to specify what kind of nonadiabatic mode we want to study and constrain. Here the situation is similar and we do hope that, in the future, the considerations reported here will serve as an inspiration for analyzing the effects of fully inhomogeneous magnetic fields on the scalar CMB anisotropies.

The analysis reported in this paper allowed us to extend the tight coupling approximation to the case when largescale magnetic fields participate in the dynamics of the plasma. The evolution equations, in the tight coupling approximation, have then been integrated numerically using, as normalization, the analytical estimate of the SachsWolfe plateau modified by the presence of large-scale magnetic fields. The reported results allow us to not only set constraints but also to include a magnetized contribution in the current strategies of parameter extraction.

From the combined analysis of the Sachs-Wolfe plateau and of the phases of Sakharov oscillations at intermediate scales, a fully inhomogeneous magnetic field uncorrelated with the adiabatic mode can be constrained to be smaller than $0.5 \mathrm{nG}$ for a slightly blue spectral slope $\varepsilon=0.1$ and for a typical comoving wave number of $1 \mathrm{Mpc}^{-1}$. The conditions implied by the SW plateau seem to be necessary but not sufficient since magnetic fields compatible with the SW bound may alter Sakharov oscillations at smaller length scales. In the most general situation, correlation angles may proliferate: there may be correlations between the magnetized contribution and the adiabatic or even nonadiabatic modes. In this case the bounds on large-scale magnetic fields become much weaker and sizable magnetic fields may be allowed.

The present analysis also shows that magnetic fields can affect the process of formation of CMB polarization. For instance, the usual approach to the Faraday rotation of CMB polarization is to assume that the polarization itself is only due to the adiabatic mode while the rotation of the polarization plane is only due to the magnetic field. This picture has been complemented by the observation that the magnetic fields affect the predecoupling initial conditions and, consequently, modify the evolution of the degree of polarization.

With the previous statements, we would not like to give the (wrong) impression that the only effect of magnetic fields on polarization power spectra arises because of the Faraday rotation effect. It could actually happen, as stressed in the Introduction, that polarization power spectra are influenced by the vector modes as well. The point is, however, that, in the standard scenario of inflationary generated fluctuations, the only primary source of polarization comes from the adiabatic mode, and the effects of 
magnetic fields, in this case, can be studied within the formalism developed in the present paper.

The tight coupling expansion is rather useful to discuss the integration of brightness perturbations in some simple cases. Moreover, it is an essential ingredient of Boltzmann codes since it is used, at early times, to avoid the integration of the (stiff) Euler equations. It will be interesting, for future studies, to analyze the space of magnetized initial conditions by solving directly the whole Boltzmann hierarchy.

Another direction for future studies would be to include consistently magnetic fields as a further parameter in the commonly employed strategies for the statistical analysis of $\mathrm{CMB}$ anisotropies. As already stressed, the present approach is a preliminary (but necessary) step for more complete numerical discussions.

\section{APPENDIX: FULLY INHOMOGENEOUS MAGNETIC FIELDS}

In the present appendix some useful details concerning the parametrization of the magnetic field correlation functions will be collected. The magnetic fields discussed here are fully inhomogeneous and are described by the twopoint function of Eq. (2.1). According to Eqs. (2.12), (2.13), and (2.14), the only two quantities determining the effects of the stochastic magnetic field background are given, respectively, by $\Omega_{\mathrm{B}}$ and $\sigma_{\mathrm{B}}$. These two variables enter both the perturbed Einstein equations and the evolution equation for the divergence of the photon-baryon velocity field, i.e., for instance Eq. (2.9). According to Eq. (2.12), the divergence of the MHD Lorentz force is given by the Laplacian of a combination of $\sigma_{\mathrm{B}}$ and $\Omega_{\mathrm{B}}$. Equation (2.12) is a simple consequence of a well-known vector identity stipulating that

$$
\partial_{i} B_{j} \partial^{j} B^{i}=\vec{\nabla} \cdot[(\vec{\nabla} \times \vec{B}) \times \vec{B}]+\frac{1}{2} \nabla^{2} B^{2} .
$$

From the definition of $\sigma_{\mathrm{B}}$ in terms of $\tilde{\Pi}_{i}^{j}$, i.e. Eq. (2.13), it is easy to show that

$$
\nabla^{2} \sigma_{\mathrm{B}}=\frac{3}{16 \pi a^{4} \rho_{\gamma}} \partial_{i} B_{j} \partial^{j} B^{i}-\frac{1}{2} \nabla^{2} \Omega_{\mathrm{B}} .
$$

Finally, using Eq. (A1) to eliminate the first term on the right-hand side of Eq. (A2) and recalling that, in MHD, the Ohmic current is solenoidal (i.e. $\vec{\nabla} \times \vec{B}=4 \pi \vec{J}$ ), Eq. (2.12) follows immediately.

Both $\Omega_{\mathrm{B}}$ and $\sigma_{\mathrm{B}}$ are quadratic in the magnetic field intensities and can also be expressed, in Fourier space, as

$$
\Omega_{\mathrm{B}}(\vec{x}, \tau)=\frac{\delta \rho_{\mathrm{B}}(\vec{x}, \tau)}{\rho_{\gamma}(\tau)}=\frac{1}{(2 \pi)^{3 / 2}} \int d^{3} q \Omega_{\mathrm{B}}(\vec{q}, \tau) e^{-i \vec{q} \cdot \vec{x}},
$$

$$
\sigma_{\mathrm{B}}(\vec{x}, \tau)=\frac{1}{(2 \pi)^{3 / 2}} \int d^{3} q e^{-i \vec{q} \cdot \vec{x}} \sigma_{\mathrm{B}}(q, \tau),
$$

with $^{7}$

$$
\begin{aligned}
\Omega_{\mathrm{B}}(\vec{q}, \tau)= & \frac{1}{(2 \pi)^{3 / 2}} \frac{1}{8 \pi \bar{\rho}_{\gamma}} \int d^{3} p B_{i}(\vec{p}) B^{i}(\vec{q}-\vec{p}), \\
\sigma_{\mathrm{B}}(\vec{q}, \tau)= & \frac{1}{(2 \pi)^{3 / 2}} \frac{1}{16 \pi \bar{\rho}_{\gamma}} \\
& \times \int d^{3} p\left[\frac{3\left(q^{j}-p^{j}\right) p^{i}}{q^{2}} B_{j}(\vec{p}) B_{i}(\vec{q}-\vec{p})\right. \\
& \left.-B_{i}(\vec{q}-\vec{p}) B^{i}(\vec{p})\right],
\end{aligned}
$$

where $\bar{\rho}_{\gamma}=a^{4}(\tau) \rho_{\gamma}(\tau)$. The correlation functions of $\Omega_{\mathrm{B}}$ and $\sigma_{\mathrm{B}}$ can then be obtained by expressing the four-field correlators in terms of the two-point functions of Eq. (2.1). In the case of $\Omega_{\mathrm{B}}$, the result of this manipulation is

$$
\begin{aligned}
&\left\langle\Omega_{\mathrm{B}}(\vec{k}, \tau) \Omega_{\mathrm{B}}(\vec{p}, \tau)\right\rangle=\frac{2 \pi^{2}}{k^{3}} \mathcal{P}_{\Omega}(k, \tau) \delta^{(3)}(\vec{k}+\vec{p}), \\
& \mathcal{P}_{\Omega}(q)= \frac{1}{4(2 \pi)^{7}} \frac{q^{3}}{\rho_{\gamma} a^{4}} \int d^{3} k\left\{1+\frac{[\vec{k} \cdot(\vec{q}+\vec{k})]^{2}}{k^{2}(\vec{q}+\vec{k})^{2}}\right\} \\
& \times P_{\mathrm{B}}(\vec{q}+\vec{k}) P_{\mathrm{B}}(\vec{k}),
\end{aligned}
$$

where, as in the bulk of the paper, $P_{\mathrm{B}}$ denotes the magnetic power spectrum appearing, for instance, in Eq. (2.1). The explicit evaluation of Eq. (A8) involves two slightly different integrals, i.e.

$$
\mathcal{W}_{1}(q)=\int_{0}^{k_{\mathrm{D}}} d k k^{\varepsilon-1} \int_{-1}^{1}\left(q^{2}+k^{2}-2 q k \lambda\right)^{(\varepsilon-3) / 2} d \lambda,
$$

$$
\begin{aligned}
\mathcal{W}_{2}(q)= & \int_{0}^{k_{\mathrm{D}}} d k k^{\varepsilon-1} \\
& \times \int_{-1}^{1}\left(q^{2}+k^{2}-2 q k \lambda\right)^{(\varepsilon-3) / 2}(q \lambda-k)^{2} d \lambda
\end{aligned}
$$

The integration over $\lambda$ (coming from the angular integral) can always be performed. In particular, in the case of Eqs. (A9) and (A10) we will have, respectively,

$$
\mathcal{W}_{1}(q)=\int_{0}^{k_{\mathrm{D}}} \frac{d \ln k}{q(\varepsilon-1)} k^{\varepsilon^{-1}}\left[|q+k|^{\varepsilon^{-1}}-|k-q|^{\varepsilon-1}\right]
$$

\footnotetext{
${ }^{7}$ The conventions on the Fourier transform are such that $B_{i}(\vec{x}, \tau)=(2 \pi)^{-3 / 2} \int d^{3} k B_{i}(\vec{k}, \tau) e^{-i \vec{k} \cdot \vec{x}}$.
} 


$$
\begin{aligned}
\mathcal{W}_{2}(q)= & \int_{0}^{k_{\mathrm{D}}} \frac{d \ln k}{q^{3}} k^{\varepsilon-1}\left\{\frac{\left[q^{2}\left(\varepsilon^{2}-2 \varepsilon-1\right)+2 k^{2}-2(\varepsilon-1) q k\right]|k+q|^{\varepsilon-1}}{(\varepsilon-1)(\varepsilon+1)(\varepsilon-3)}\right. \\
& \left.-\frac{\left[q^{2}\left(\varepsilon^{2}-2 \varepsilon-1\right)+2 k^{2}+2(\varepsilon-1) q k\right]|k-q|^{\varepsilon-1}}{(\varepsilon-1)(\varepsilon+1)(\varepsilon-3)}\right\} .
\end{aligned}
$$

The integration over $k$ is performed between 0 and the diffusion momentum $k_{\mathrm{D}}$ assuming that the magnetic field spectrum is nearly scale invariant, i.e. $0<\varepsilon<1$. In this regime, it is useful to break the integral between 0 and $k_{\mathrm{D}}$ as $\int_{0}^{k_{\mathrm{D}}}[\ldots] d k=\int_{0}^{q}[\ldots] d k+\int_{q}^{k_{\mathrm{D}}}[\ldots] d k$. In the interval $[0, q]$ we can then neglect the higher orders in $|k / q|$ appearing in the integrand. In the interval $\left[q, k_{\mathrm{D}}\right]$ we can similarly neglect the higher orders in $|q / k|$. This observation allows us to express the integrals $\mathcal{W}_{1}$ and $\mathcal{W}_{2}$ in terms of simple powers. Using this approach it can be easily shown that the upper limit of integration, i.e. $k_{\mathrm{D}}$, does not contribute to the final result if the magnetic field spectrum is slightly blue (i.e. $\varepsilon>0$ ) as assumed throughout the paper. The final result is then

$$
\begin{gathered}
\mathcal{P}_{\Omega}(k)=\mathcal{F}(\varepsilon) \bar{\Omega}_{\mathrm{B} L}^{2}\left(\frac{k}{k_{L}}\right)^{2 \varepsilon}, \\
\mathcal{F}(\varepsilon)=\frac{(6-\varepsilon)(2 \pi)^{2 \varepsilon}}{\varepsilon(3-2 \varepsilon) \Gamma^{2}(\varepsilon / 2)}, \\
\bar{\Omega}_{\mathrm{B} L}=\frac{\rho_{\mathrm{B} L}}{\bar{\rho}_{\gamma}}, \quad \rho_{\mathrm{B} L}=\frac{B_{L}^{2}}{8 \pi} .
\end{gathered}
$$

The correlation function of $\sigma_{\mathrm{B}}$ can be expressed as

$$
\left\langle\sigma_{\mathrm{B}}(\vec{q}, \tau) \sigma_{\mathrm{B}}(\vec{k}, \tau)\right\rangle=\frac{2 \pi^{2}}{q^{3}} \mathcal{P}_{\sigma}(q, \tau) \delta^{(3)}(\vec{q}+\vec{k}),
$$

where

$$
\begin{aligned}
\mathcal{P}_{\sigma}(q)= & \frac{1}{(2 \pi)^{7}} \frac{q^{3}}{\bar{\rho}_{\gamma}} \int d^{3} k P_{\mathrm{B}}(k) P_{\mathrm{B}}(\vec{q}+\vec{k}) \\
& \times\left\{1+\frac{[\vec{k} \cdot(\vec{k}+\vec{q})]^{2}}{k^{2}|\vec{k}+\vec{q}|^{2}}\right. \\
& -\frac{6}{q^{2}}\left[\vec{k} \cdot(\vec{k}+\vec{q})-\frac{[\vec{k} \cdot(\vec{q}+\vec{k})]^{3}}{k^{2}|\vec{k}+\vec{q}|^{2}}\right] \\
& +\frac{9}{q^{4}}\left[k^{2}|\vec{k}+\vec{q}|^{2}-2[\vec{k} \cdot(\vec{k}+\vec{q})]^{2}\right. \\
& \left.\left.+\frac{[\vec{k} \cdot(\vec{q}+\vec{k})]^{4}}{k^{2}|\vec{q}+\vec{k}|^{2}}\right]\right\} .
\end{aligned}
$$

In this second case, following the same strategy described in connection with Eqs. (A11) and (A12), the result of the integrations can be written as

$$
\begin{aligned}
\mathcal{P}_{\sigma}(k) & =G(\varepsilon) \bar{\Omega}_{\mathrm{B} L}^{2}\left(\frac{k}{k_{L}}\right)^{2 \varepsilon}, \\
G(\varepsilon) & =\frac{4\left(188-4 \varepsilon^{2}-66 \varepsilon\right)(2 \pi)^{2 \varepsilon}}{3 \varepsilon(3-\varepsilon)(2 \varepsilon+1) \Gamma^{2}(\varepsilon / 2)} .
\end{aligned}
$$

As previously remarked, it has been assumed that the magnetic spectrum is nearly scale-invariant (i.e. $\varepsilon<1$ ) but slightly blue (i.e. $\varepsilon>0$ ).
[1] H. V. Peiris et al. (WMAP Collaboration), Astrophys. J. Suppl. Ser. 148, 213 (2003).

[2] L. Page et al. (WMAP Collaboration), astro-ph/0603450.

[3] D. N. Spergel et al. (WMAP Collaboration), astro-ph/ 0603449.

[4] P. J.E. Peebles and J.T. Yu, Astrophys. J. 162, 815 (1970).

[5] A. G. Doroshkevich, Ya. B. Zeldovich, and R. A. Sunyaev, Sov. Astron. 22, 523 (1978).

[6] M. Zaldarriaga and D. D. Harari, Phys. Rev. D 52, 3276 (1995).

[7] M. Giovannini, Phys. Rev. D 73, 101302 (2006).

[8] A. Kosowsky and A. Loeb, Astrophys. J. 469, 1 (1996).

[9] M. Giovannini, Phys. Rev. D 56, 3198 (1997).

[10] C. Scoccola, D. Harari, and S. Mollerach, Phys. Rev. D 70, 063003 (2004).

[11] A. Kosowsky, T. Kahniashvili, G. Lavrelashvili, and B. Ratra, Phys. Rev. D 71, 043006 (2005).
[12] L. Campanelli, A. D. Dolgov, M. Giannotti, and F. L. Villante, Astrophys. J. 616, 1 (2004).

[13] M. Giovannini, Phys. Rev. D 71, 021301 (2005).

[14] K. Subramanian, astro-ph/0601570.

[15] M. Giovannini, Classical Quantum Gravity 23, R1 (2006).

[16] R. Beck, A. Brandenburg, D. Moss, A. Shukurov, and D. Sokoloff, Annu. Rev. Astron. Astrophys. 34, 155 (1996).

[17] R. Beck and B. M. Gaensler, New Astron. Rev. 48, 1289 (2004); R. Beck, astro-ph/0512501.

[18] B. M. Gaensler, R. Beck, and L. Feretti, New Astron. Rev. 48, 1003 (2004).

[19] F. Govoni and L. Feretti, Int. J. Mod. Phys. D 13, 1549 (2004).

[20] L. Feretti, E. Orru, G. Brunetti, G. Giovannini, N. Kassim, and G. Setti, Astron. Astrophys. 423, 111 (2004).

[21] T.E. Clarke, P.P. Kronberg, and H. Boehringer, Astrophys. J. 547, L111 (2001).

[22] P. P. Kronberg, Rep. Prog. Phys. 57, 325 (1994). 
[23] P. P. Kronberg, H. Lesch, and U. Hopp, Astrophys. J. 511, 56 (1999).

[24] Y. Xu, P.P. Kronberg, S. Habib, and Q. W. Dufton, Astrophys. J. 637, 19 (2006).

[25] J. L. Han, K. Ferriere, and R. N. Manchester, Astrophys. J. 610, 820 (2004).

[26] J. L. Han, R. N. Manchester, A. G. Lyne, G. J. Qiao, and W. van Straten, Astrophys. J. 642, 868 (2006).

[27] M. Haverkorn, B. M. Gaensler, J.C. Brown, N. S. Bizunok, N. M. McClure-Griffiths, J.M. Dickey, and A. J. Green, Astrophys. J. 637, L33 (2006).

[28] M. Giovannini, Int. J. Mod. Phys. D 13, 391 (2004).

[29] A. Brandenburg and K. Subramanian, Phys. Rep. 417, 1 (2005).

[30] R. M. Kulsrud, Annu. Rev. Astron. Astrophys. 37, 37 (1999).

[31] E. T. Vishniac, A. Lazarian, and J. Cho, Lect. Notes Phys. 614, 376 (2003).

[32] G. Chen et al., Astrophys. J. 611, 655 (2004).

[33] P. D. Naselsky, L. Y. Chiang, P. Olesen, and O. V. Verkhodanov, Astrophys. J. 615, 45 (2004).

[34] A. Hajian and T. Souradeep, astro-ph/0501001.

[35] K. Subramanian and J.D. Barrow, Phys. Rev. Lett. 81, 3575 (1998).

[36] K. Subramanian and J. D. Barrow, Mon. Not. R. Astron. Soc. 335, L57 (2002).

[37] K. Subramanian, T. R. Seshadri, and J. D. Barrow, Mon. Not. R. Astron. Soc. 344, L31 (2003).

[38] T. Seshadri and K. Subramanian, Phys. Rev. Lett. 87, 101301 (2001); Phys. Rev. D 72, 023004 (2005).

[39] A. Mack, T. Kahniashvili, and A. Kosowsky, Phys. Rev. D 65, 123004 (2002).

[40] A. Lewis, Phys. Rev. D 70, 043518 (2004).

[41] T. Kahniashvili and B. Ratra, Phys. Rev. D 71, 103006 (2005).

[42] C. G. Tsagas and J.D. Barrow, Classical Quantum Gravity 15, 3523 (1998); C. Tsagas and R. Maartens, Phys. Rev. D 61, 083519 (2000).

[43] M. Giovannini, Phys. Rev. D 70, 123507 (2004).

[44] Y. Zeldovich, Sov. Phys. JETP 21, 656 (1965).

[45] Y. Zeldovich and I. Novikov, The Structure and Evolution of the Universe (Chicago University Press, Chicago Illinois, 1971).

[46] E. Harrison, Rev. Mod. Phys. 39, 862 (1967).

[47] J. M. Bardeen, Phys. Rev. D 22, 1882 (1980).

[48] T. J. M. Boyd and J. J. Sanderson, The Physics of Plasmas (Cambridge University Press, Cambridge, UK, 2003).

[49] D. Biskamp, Non-linear Magnetohydrodynamics (Cambridge University Press, Cambridge, UK, 1994).

[50] A. G. Riess et al., Astrophys. J. 607, 665 (2004).

[51] P. Astier et al., Astron. Astrophys. 447, 31 (2006).

[52] P. Olesen, Phys. Lett. B 398, 321 (1997).

[53] A. Brandenburg, K. Enqvist, and P. Olesen, Phys. Lett. B 392, 395 (1997).

[54] M. Giovannini, Phys. Rev. D 71, 021301 (2005).

[55] W. Hu and N. Sugiyama, Astrophys. J. 444, 489 (1995); 471, 30 (1996).

[56] C.-P. Ma and E. Bertschinger, Astrophys. J. 455, 7 (1995).

[57] M. Giovannini, Classical Quantum Gravity 23, 4991 (2006).

[58] J. Bardeen, P. Steinhardt, and M. Turner, Phys. Rev. D 28, 679 (1983).

[59] R. Brandenberger, R. Kahn, and W. Press, Phys. Rev. D 28, 1809 (1983).

[60] B. Ratra, Astrophys. J. 391, L1 (1992).

[61] M. Gasperini, M. Giovannini, and G. Veneziano, Phys. Rev. Lett. 75, 3796 (1995).

[62] A. Challinor and A. Lasenby, Phys. Rev. D 58, 023001 (1998).

[63] M. Bucher, K. Moodley, and N. Turok, Phys. Rev. D 62, 083508 (2000).

[64] K. Enqvist, H. Kurki-Suonio, and J. Valiviita, Phys. Rev. D 62, 103003 (2000).

[65] J. Valiviita and V. Muhonen, Phys. Rev. Lett. 91, 131302 (2003).

[66] H. Kurki-Suonio, V. Muhonen, and J. Valiviita, Phys. Rev. D 71, 063005 (2005).

[67] S. Chandrasekar, Radiative Transfer (Dover, New York, 1966).

[68] H. Jorgensen, E. Kotok, P. Naselsky, and I. Novikov, Astron. Astrophys. 294, 639 (1995). 